\title{
Inclusive jet spectrum for small-radius jets
}

\author{
Mrinal Dasgupta, ${ }^{a}$ Frédéric A. Dreyer, ${ }^{b, c, d}$ Gavin P. Salam ${ }^{d, 1}$ and Gregory Soyez ${ }^{e}$ \\ ${ }^{a}$ Consortium for Fundamental Physics, School of Physics $\&$ Astronomy, University of Manchester, \\ Manchester M13 9PL, U.K. \\ ${ }^{b}$ Sorbonne Universités, UPMC Univ Paris 06, UMR 7589, LPTHE, \\ F-75005, Paris, France \\ ${ }^{c}$ CNRS, UMR 7589, LPTHE, \\ F-75005, Paris, France \\ ${ }^{d}$ CERN, Theoretical Physics Department, \\ CH-1211 Geneva 23, Switzerland \\ ${ }^{e}$ IPhT, CEA Saclay, CNRS UMR 3681, \\ F-91191 Gif-sur-Yvette, France \\ E-mail: Mrinal.Dasgupta@manchester.ac.uk, dreyer@lpthe.jussieu.fr, \\ gavin.salam@cern.ch, gregory.soyez@cea.fr
}

ABSTRACT: Following on our earlier work on leading-logarithmic $\left(\mathrm{LL}_{R}\right)$ resummations for the properties of jets with a small radius, $R$, we here examine the phenomenological considerations for the inclusive jet spectrum. We discuss how to match the NLO predictions with small- $R$ resummation. As part of the study we propose a new, physically-inspired prescription for fixed-order predictions and their uncertainties. We investigate the $R$-dependent part of the next-to-next-to-leading order (NNLO) corrections, which is found to be substantial, and comment on the implications for scale choices in inclusive jet calculations. We also examine hadronisation corrections, identifying potential limitations of earlier analytical work with regards to their $p_{t}$-dependence. Finally we assemble these different elements in order to compare matched $(\mathrm{N}) \mathrm{NLO}+\mathrm{LL}_{R}$ predictions to data from ALICE and ATLAS, finding improved consistency for the $R$-dependence of the results relative to NLO predictions.

KEYWORDS: Jets, QCD Phenomenology

ARXiv EPrint: 1602.01110

\footnotetext{
${ }^{1}$ On leave from CNRS, UMR 7589, LPTHE, F-75005, Paris, France.
} 


\section{Contents}

1 Introduction 1

2 Small- $R$ resummation for the inclusive jet spectrum $\quad 3$

2.1 Recall of the small- $R$ resummation formalism at $L_{R}$ accuracy 3

2.2 Range of validity of the small- $R$ approximation and effects beyond $\mathrm{LL}_{R} \quad 5$

3 Matching NLO and $\mathrm{LL}_{R} \quad 7$

$\begin{array}{ll}3.1 \text { Matching prescriptions } & 7\end{array}$

$\begin{array}{lll}3.2 & \text { Unphysical cancellations in scale dependence } & 8\end{array}$

$\begin{array}{lll}3.3 & \mathrm{NLO}+\mathrm{LL}_{R} \text { matched results } & 9\end{array}$

4 Matching to NNLO 11

4.1 Matching prescription 11

$\begin{array}{ll}4.2 & \text { A stand-in for NNLO: } \mathrm{NNLO}_{R} \\ & 12\end{array}$

$\begin{array}{lll}4.3 & \text { Results at } \mathrm{NNLO}_{R} \text { and } \mathrm{NNLO}_{R}+\mathrm{LL}_{R} & 13\end{array}$

$\begin{array}{lll}4.4 & \text { Impact of finite two-loop corrections } & 14\end{array}$

$\begin{array}{ll}\text { 4.5 Comparison to POWHEG } & 17\end{array}$

5 Hadronisation $\quad 19$

6 Comparisons to data 23

6.1 Comparison to ALICE data 24

6.2 Comparison to ATLAS data 28

6.3 Brief comparisons with an NNLO $K$-factor 31

7 Conclusion $\quad 32$

A Differences in $\alpha_{s}$ and $t$ expansions $\quad 35$

B Scale choice beyond leading order $\quad 36$

\section{Introduction}

Jets are used in a broad range of physics analyses at colliders and notably at CERN's large hadron collider (LHC). The study of jets requires the use of a jet definition and for many of the algorithms in use today that jet definition involves a "radius" parameter, $R$, which determines how far in angle a jet clusters radiation. A limit of particular interest is that where $R$ is taken small. One reason is that contamination from multiple simultaneous $p p$ interactions ("pileup") is minimised, as is the contribution from the large underlying event 
when studying heavy-ion collisions. Furthermore small- $R$ subjets are often a component of jet substructure analyses used to reconstruct hadronic decays of highly boosted weak bosons and top quarks. Finally a powerful systematic cross-check in precision jet studies comes from the variation of $R$, e.g. to verify that conclusions about data-theory agreement remain stable. Such a variation will often bring one into the small- $R$ limit.

In the small- $R$ limit the perturbative series involves terms $\alpha_{s}^{n} \ln ^{n} 1 / R^{2}$, where the smallness of $\alpha_{s}^{n}$ is partially compensated by large logarithms $\ln ^{n} 1 / R^{2}$. The first order $\alpha_{s} \ln 1 / R^{2}$ terms (together with the constant) were first calculated long ago [1-3] and have been examined also more recently [4-6]. About a year ago, the whole tower of leadinglogarithmic $\left(\mathrm{LL}_{R}\right)$ terms was determined [7], i.e. $\alpha_{s}^{n} \ln ^{n} 1 / R^{2}$ for all $n$, for a range of observables (for related work, see also refs. $[8,9]$ ). Work is also ongoing towards next-toleading logarithmic accuracy, $\mathrm{NLL}_{R}[10,11]$, however the concrete results do not yet apply to hadron-collider jet algorithms.

From the point of view of phenomenological studies, there has so far been one investigation of the impact of small- $R$ resummation in the context of jet vetoes in Higgs production [12]. Though small- $R$ contributions have a significant impact on the results, most of their effect (at the phenomenologically relevant $R$ value of 0.4 ) is already accounted for in fixed-order and $p_{t}$-resummed calculations. Accordingly the resummation brings only small additional changes.

In this article, we examine the phenomenological impact of small- $R$ terms for the archetypal hadron-collider jet observable, namely the inclusive jet spectrum. This observable probes the highest scales that are accessible at colliders and is used for constraining parton distribution functions (PDFs), determining the strong coupling and also in studies of hard probes in heavy-ion collisions. Two factors can contribute to enhance small- $R$ effects in the inclusive jet spectrum: firstly, it falls steeply as a function of $p_{t}$, which magnifies the impact of any physical effect that modifies the jet's energy. Secondly, a wide range of $R$ values has been explored for this observable, going down as far as $R=0.2$ in measurements in proton-proton collisions by the ALICE collaboration [13]. That wide range of $R$ has been exploited also in studies of ratios of cross sections at different $R$ values [13-17]. For $R=0.2$, $\mathrm{LL}_{R}$ small- $R$ effects can be responsible for up to $40 \%$ modifications of the jet spectrum.

A first part of our study (section 2) will be to establish the region of $R$ where the small- $R$ approximation is valid and to examine the potential impact of effects beyond the $\mathrm{LL}_{R}$ approximation. This will point to the need to include at the least the subleading $R$-enhanced terms that arise at next-to-next-to-leading order (NNLO) and motivate us to devise schemes to match $\mathrm{LL}_{R}$ resummation with both NLO and NNLO calculations (sections 3 and 4 respectively). At NLO we will see indications of spuriously small scale dependence and discuss how to resolve the issue. Concerning NNLO, since the full calculation is work in progress $[18,19]$, to move forwards we will introduce an approximation that we refer to as " $\mathrm{NNLO}_{R}$ ". It contains the full $R$-dependence of the NNLO prediction but misses an $R$-independent, though $p_{t}$-dependent, constant term. By default we will take it to be zero at some reference radius $R_{\mathrm{m}}$, but we will also examine the impact of other choices.

In addition to the perturbative contributions at small- $R$, one must take into account non-perturbative effects, which are enhanced roughly as $1 / R$ at small $R$ and grow large 
especially at smaller values of $p_{t}$. Two approaches exist for incorporating them, one based on analytic calculations [20], the other based on the differences between parton and hadronlevel results in Monte Carlo event generators such as Pythia [21, 22] and Herwig [23-26]. Based on our studies in section 5, we adopt the Monte Carlo approach for our comparisons to data. These are the subject of section 6 , where we examine data from the ALICE collaboration [13] at $R=0.2$ and 0.4 , and from the ATLAS [27] collaboration at $R=0.4$ and 0.6.

A broad range of dynamically-generated plots comparing different theory predictions across a range of rapidities, transverse momenta and $R$ values can be viewed online [28]. Furthermore some of the plots included in the arXiv source for this paper contain additional information in the form of extra pages not displayed in the manuscript.

\section{Small- $R$ resummation for the inclusive jet spectrum}

\subsection{Recall of the small- $R$ resummation formalism at $L L_{R}$ accuracy}

As for the inclusive hadron spectrum [29], the small- $R$ inclusive "microjet" spectrum can be obtained [7] from the convolution of the leading-order inclusive spectrum of partons of flavour $k$ and transverse momentum $p_{t}^{\prime}, \frac{d \sigma^{(k)}}{d p_{t}^{\prime}}$, with the inclusive microjet fragmentation function, $f_{\text {jet } / k}^{\text {incl }}\left(p_{t} / p_{t}^{\prime}, t\right)$, for producing microjets carrying a fraction $p_{t} / p_{t}^{\prime}$ of the parton's momentum,

$$
\sigma^{\mathrm{LL}_{R}}\left(p_{t}, R\right) \equiv \frac{d \sigma_{\mathrm{jet}}^{\mathrm{LL}}}{d p_{t}}=\sum_{k} \int_{p_{t}} \frac{d p_{t}^{\prime}}{p_{t}^{\prime}} f_{\mathrm{jet} / k}^{\mathrm{incl}}\left(\frac{p_{t}}{p_{t}^{\prime}}, t\left(R, R_{0}, \mu_{R}\right)\right) \frac{d \sigma^{(k)}}{d p_{t}^{\prime}} .
$$

To keep the notation compact, we use $\sigma^{\mathrm{LL}_{R}}\left(p_{t}, R\right)$ to denote either a differential cross section, or the cross section in a given $p_{t}$ bin, depending on the context. At $\mathrm{LL}_{R}$ accuracy, the small- $R$ effects are entirely contained in the fragmentation function, which depends on $R$ through the evolution variable $t$, defined as

$$
t\left(R, R_{0}, \mu_{R}\right)=\int_{R^{2}}^{R_{0}^{2}} \frac{d \theta^{2}}{\theta^{2}} \frac{\alpha_{s}\left(\mu_{R} \theta / R_{0}\right)}{2 \pi}=\frac{1}{b_{0}} \ln \frac{1}{1-\frac{\alpha_{s}\left(\mu_{R}\right)}{2 \pi} b_{0} \ln \frac{R_{0}^{2}}{R^{2}}},
$$

with $b_{0}=\frac{11 C_{A}-4 T_{R} n_{f}}{6} .{ }^{1}$ Here, $R_{0}$ is the angular scale, of order 1 , at which the small-radius approximation starts to become valid. For $R=R_{0}$, or equivalently $t=0$, the fragmentation function has the initial condition $f_{\text {jet } / k}^{\text {incl }}(z, 0)=\delta(1-z)$. It can be determined for other $t$ values by solving a DGLAP-like evolution equation in $t$ [7]. These results are identical for any standard hadron-collider jet algorithm of the generalised- $k_{t}$ [30-34] and SISCone [35] families, with differences between them appearing only at subleading logarithmic order. In this work we will restrict our attention to the anti- $k_{t}$ algorithm (as implemented in FastJet v3.1.3 [36]). The $\mathrm{LL}_{R}$ resummation is implemented with the aid of HOPPET [37].

\footnotetext{
${ }^{1}$ The choice of whether to use $\alpha_{s}\left(\mu_{R} \theta / R_{0}\right)$ or $\alpha_{s}\left(\mu_{R} \theta\right)$ in the integral is arbitrary. We choose the former because it ensures that $\alpha_{s}\left(\mu_{R}\right)$ factorises from the logarithm of $R$ in the right-hand side.
} 


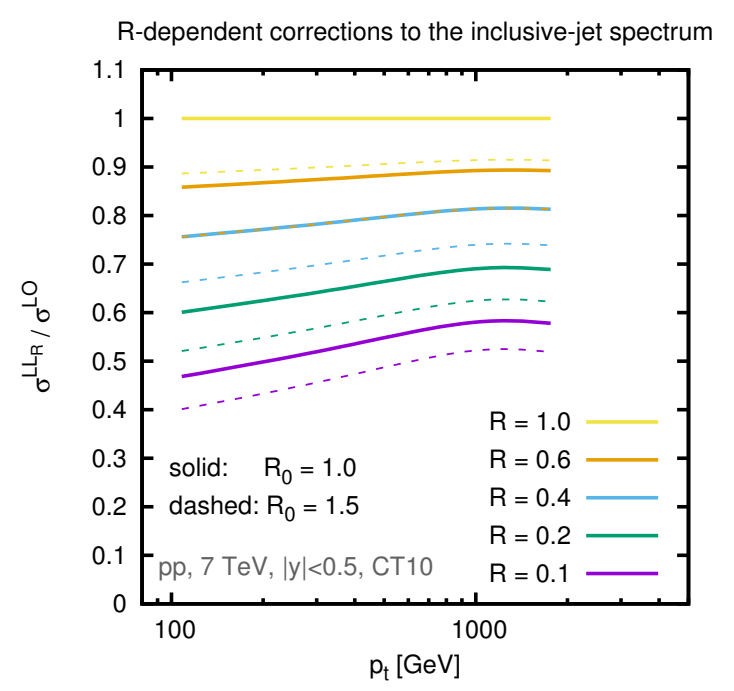

Figure 1. Impact of $R$-dependent terms in the inclusive-jet spectrum, illustrated using the small- $R$ resummation factor obtained from the ratio of $\sigma^{\mathrm{LL}_{R}}$ in eq. (2.1) to the leading order inclusive jet spectrum $\sigma^{\text {LO }}$. It is shown as a function of the jet $p_{t}$ for different jet radius values. For each $R$ value, the plot illustrates the impact of two choices of $R_{0}: R_{0}=1$ (our default) as solid lines and $R_{0}=1.5$ as dashed lines.

The phenomenological relevance of the small- $R$ terms is illustrated in figure 1, which shows the ratio of the jet spectrum with small- $R$ resummation effects to the LO jet spectrum. ${ }^{2}$ For this and a number of later plots, the $p_{t}$ and rapidity ranges and the collider energy choice correspond to those of ATLAS measurements [27], to which we will later compare our results. We show the impact of resummation for a range of $R$ values from 0.1 to 1.0 and two $R_{0}$ choices. The smallest $R$ values typically in use experimentally are in the range $0.2-0.4$ and one sees that the fragmentation of partons into jets brings up to $40 \%$ reduction in the cross section for the smaller of these radii. The fact that the small- $R$ effects are substantial is one of the motivations for our work here.

From the point of view of phenomenological applications, the question that perhaps matters more is the impact of corrections beyond NLO (or forthcoming NNLO), since fixed order results are what are most commonly used in comparisons to data. This will be most easily quantifiable when we discuss matched results in sections 3 and 4 . Note that there was already some level of discussion of effects beyond fixed order in ref. [7], in terms of an expansion in powers of $t$. However comparisons to standard fixed order refer to an expansion in $\alpha_{s}$, which is what we will be using throughout this article. A brief discussion of the different features of $t$ and $\alpha_{s}$ expansions is given in appendix $\mathrm{A}$.

\footnotetext{
${ }^{2}$ Although for this purpose we have used the small- $R$ resummed calculations, we could also have used NLO calculations which would also indicate similarly visible $R$-dependent effects. The differences between the small- $R$ resummation and NLO predictions will be discussed later.
} 


\subsection{Range of validity of the small- $R$ approximation and effects beyond $L_{R}$}

In order to carry out a reliable phenomenological study of small- $R$ effects it is useful to ask two questions about the validity of our $\mathrm{LL}_{R}$ small- $R$ approach. Firstly we wish to know from what radii the underlying small-angle approximation starts to work. Secondly, we want to determine the potential size of small- $R$ terms beyond $\mathrm{LL}_{R}$ accuracy.

To investigate the first question we take the full next-to-leading-order (NLO) calculation for the inclusive jet spectrum from the NLOJet++ program [38], and look at the quantity $\Delta_{1}\left(p_{t}, R, R_{\text {ref }}\right)$, where

$$
\Delta_{i}\left(p_{t}, R, R_{\mathrm{ref}}\right) \equiv \frac{\sigma_{i}\left(p_{t}, R\right)-\sigma_{i}\left(p_{t}, R_{\mathrm{ref}}\right)}{\sigma_{0}\left(p_{t}\right)} .
$$

Here $\sigma_{i}\left(p_{t}\right)$ corresponds to the order $\alpha_{s}^{2+i}$ contribution to the inclusive jet cross section in a given bin of $p_{t}$. This can be compared to a similar ratio, $\Delta_{1}^{\mathrm{LL}_{R}}\left(p_{t}, R, R_{\mathrm{ref}}\right)$, obtained from the NLO expansion of eq. (2.1) instead of the exact NLO result. ${ }^{3}$ The quantity $R_{\text {ref }}$ here is some small reference radius at which one expects the small- $R$ approximation to be valid; we choose $R_{\text {ref }}=0.1$. Figure 2 (left) shows the comparison of $\Delta_{1}$ (filled squares) and $\Delta_{1}^{\mathrm{LL}_{R}}$ (crosses) as a function of $p_{t}$ for several different $R$ values. One sees very good agreement between $\Delta_{1}$ and $\Delta_{1}^{\mathrm{LL}_{R}}$ for the smaller $R$ values, while the agreement starts to break down for $R$ in the vicinity of $1-1.5$. This provides grounds for using the small- $R$ approximation for $R$ values $\lesssim 0.6$ and motivates a choice of $R_{0}$ in range $1-1.5$. We will take $R_{0}=1$ as our default, and use $R_{0}=1.5$ as a probe of resummation uncertainties.

Next let us examine effects of subleading small- $R$ logarithms, terms that come with a factor $\alpha_{s}^{n} \ln ^{n-1} R$ relative to the Born cross section. While there has been some work investigating such classes of terms in refs. $[10,11]$, those results do not apply to hadroncollider jet algorithms. Instead, here we examine the $R$ dependence in the NNLO part of the inclusive jet cross section to evaluate the size of these terms. Because the $R$ dependence starts only at order $\alpha_{s}^{3}$, we can use the NLO 3-jet component of the NLOJet++ program to determine these terms. More explicitly, we use the fact that

$$
\sigma^{\mathrm{NNLO}}(R)-\sigma^{\mathrm{NNLO}}\left(R_{\text {ref }}\right)=\sigma^{\mathrm{NLO}_{3 j}}(R)-\sigma^{\mathrm{NLO}_{3 j}}\left(R_{\text {ref }}\right) .
$$

To determine this difference in practice, for each event in the NLOJet++ 3-jet run we apply the following procedure: we cluster the event with radius $R$ and for each resulting jet add the event weight to the jet's corresponding $p_{t}$ bin; we then recluster the particles with radius $R_{\text {ref }}$ and for each jet subtract the event weight from the corresponding $p_{t}$ bin. For this procedure to give a correct answer, it is crucial not to have any 3 -jet phasespace cut in the NLO 3-jet calculation (i.e. there is no explicit requirement of a 3 rd jet). ${ }^{4}$

\footnotetext{
${ }^{3} \Delta_{1}^{\mathrm{LL}_{R}}\left(p_{t}, R, R_{\mathrm{ref}}\right)$ is independent of $R_{0}$ because the $R_{0}$ cancels between the two terms in the numerator.

${ }^{4}$ Note that we have encountered issues with the convergence of the NLOJet++ calculation, with some bins showing extremely large excursions in individual runs. To obtain stable results, we perform a combination of a large number of runs (order 2000-4000) in which each bin's weight from a given run is inversely proportional to the square of its statistical error. Given that such weighted combinations are known to give biased results, we then apply a global correction factor to $\Delta_{1+2}\left(p_{t}, R, R_{\text {ref }}\right)$ across all bins. That factor
} 

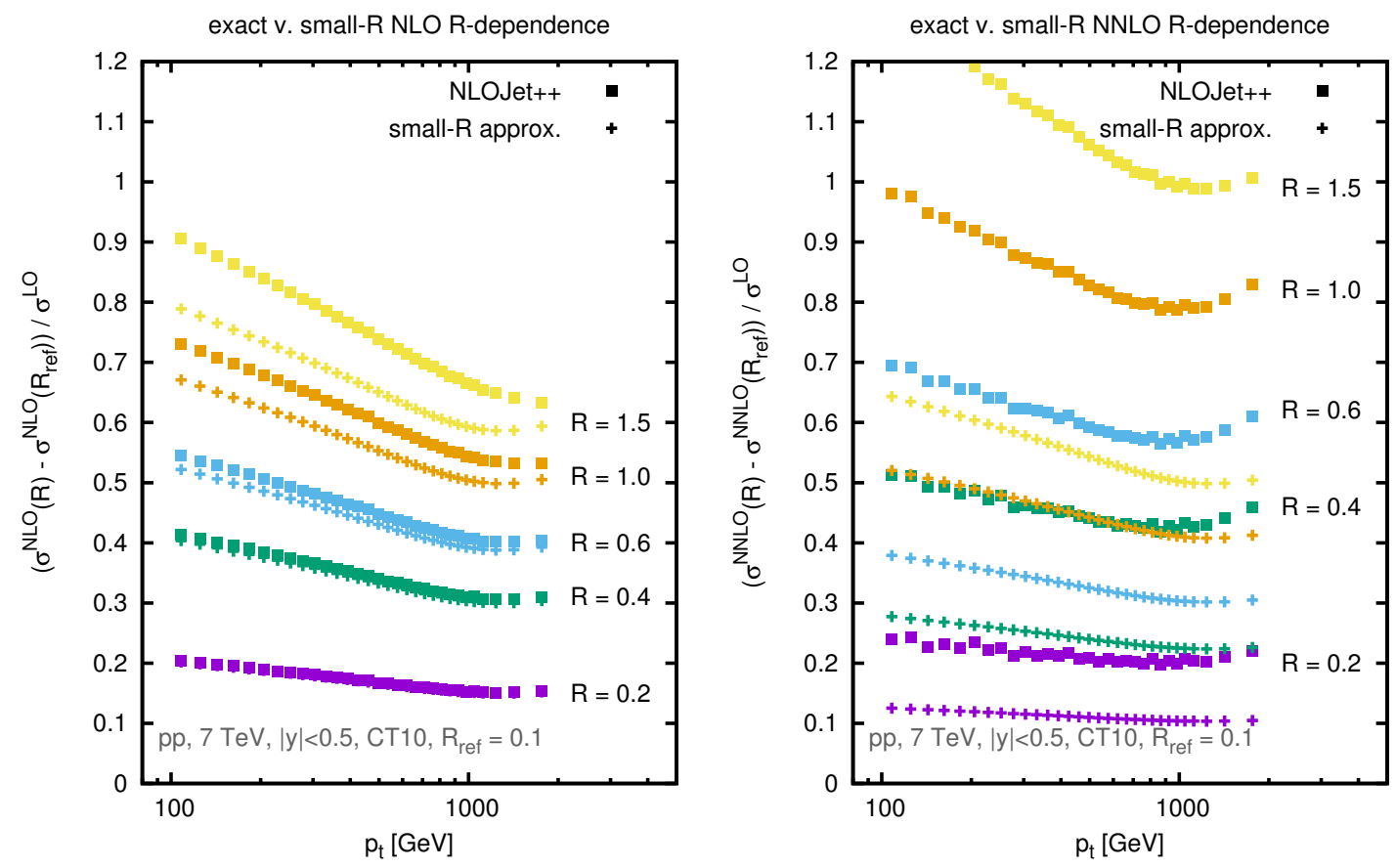

Figure 2. Left: comparison of the $R$ dependence in the exact and small- $R$ approximated NLO expansion, using eq. (2.3), shown as a function of jet transverse momentum $p_{t}$, for $\sqrt{s}=7 \mathrm{TeV}$ in the rapidity region $|y|<0.5$. Right: comparison of $\Delta_{1+2}\left(p_{t}, R, R_{\text {ref }}\right)$ and $\Delta_{1+2}^{\mathrm{LL}_{R}}\left(p_{t}, R, R_{\text {ref }}\right)$ (cf. eq. (2.5)). In both plots CT10 NLO PDFs [39] are used, while the renormalisation and factorisation scales are set equal to the $p_{t}$ of the highest- $p_{t} R=1$ jet in the event (this same scale is used for all $R$ choices in the final jet finding).

Hence, we can then examine

$$
\Delta_{1+2}\left(p_{t}, R, R_{\mathrm{ref}}\right) \equiv \Delta_{1}\left(p_{t}, R, R_{\mathrm{ref}}\right)+\Delta_{2}\left(p_{t}, R, R_{\mathrm{ref}}\right)
$$

and its corresponding $\mathrm{LL}_{R}$ approximation, $\Delta_{1+2}^{\mathrm{LL}_{R}}\left(p_{t}, R, R_{\text {ref }}\right)$. The reason for including both NLO and NNLO terms is to facilitate comparison of the size of the results with that of the pure NLO piece. The results for $\Delta_{1+2}$ (filled squares) and $\Delta_{1+2}^{\mathrm{LL}_{R}}$ (crosses) are shown in figure 2 (right). The difference between the crosses in the left-hand and right-hand plots is indicative of the size of the NNLO LL $R$ contribution. At small $R$, the difference between the crosses and solid squares in the right-hand plot gives the size of the $\mathrm{NLL}_{R}$ contribution at NNLO. It is clear that this is a substantial contribution, of the same order of magnitude as the $\mathrm{LL}_{R}$ contribution itself, but with the opposite sign. Ideally one would therefore carry out a full $\mathrm{NLL}_{R}$ calculation. That, however, is beyond the scope of this article.

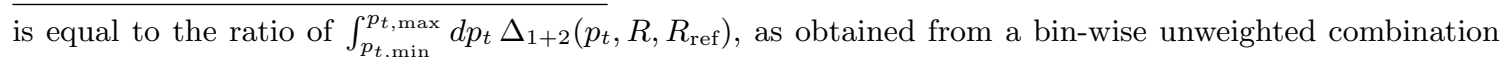
(with removal of a few percent of outlying runs in each bin) and a bin-wise weighted combination (an alternative approach to a similar issue was recently discussed in ref. [40]). We believe that the systematics associated with this procedure are at the level of a couple of percent. 
Instead we will include a subset of the subleading $\ln R$ terms by combining the $\mathrm{LL}_{R}$ resummation with the exact $R$ dependence up to NNLO fixed order, i.e. the terms explicitly included in the solid squares in figure 2.

\section{Matching $\mathrm{NLO}$ and $\mathrm{LL}_{R}$}

For phenomenological predictions, it is necessary to combine the $\mathrm{LL}_{R}$ resummation with results from fixed-order calculations. In this section we will first examine how to combine $\mathrm{LL}_{R}$ and NLO results, and then proceed with a discussion of NNLO corrections.

\subsection{Matching prescriptions}

One potential approach for combining $\mathrm{LL}_{R}$ and NLO results would be to use an additive type matching,

$$
\sigma^{\mathrm{NLO}+\mathrm{LL}_{R}, \text { add. }}(R)=\sigma^{\mathrm{LL}_{R}}(R)+\sigma_{1}(R)-\sigma_{1}^{\mathrm{LL}_{R}}(R),
$$

where $\sigma_{1}(R)$ denotes the pure NLO contribution to the inclusive jet spectrum (without the LO part, as in section 2.2) and $\sigma_{1}^{\mathrm{LL}_{R}}(R)$ refers to the pure NLO contribution within the $\mathrm{LL}_{R}$ resummation. For compactness, the $p_{t}$ argument in the cross sections has been left implicit.

A simple, physical condition that the matching must satisfy is that in the limit $R \rightarrow 0$, the ratio of the matched result to the LO result should tend to zero perturbatively, ${ }^{5}$

$$
\frac{\sigma^{\mathrm{NLO}+\mathrm{LL}_{R}}}{\sigma_{0}} \rightarrow 0 \quad \text { for } R \rightarrow 0
$$

Eq. (3.1) does not satisfy this property: while $\sigma^{\mathrm{LL}_{R}} / \sigma_{0}$ does tend to zero, the quantity $\left(\sigma_{1}-\sigma_{1}^{\mathrm{LL}}{ }_{R}\right) / \sigma_{0}$ instead tends to a constant for small $R$. We will therefore not use additive matching.

Another class of matching procedure is multiplicative matching. One simple version of multiplicative matching is given by

$$
\sigma^{\mathrm{NLO}+\mathrm{LL}_{R}, \text { mult.simple }}=\frac{\sigma^{\mathrm{LL}_{R}}(R)}{\sigma_{0}} \times\left(\sigma_{0}+\sigma_{1}(R)-\sigma_{1}^{\mathrm{LL}_{R}}(R)\right)
$$

Because $\sigma_{1}^{\mathrm{LL}_{R}}(R)$ contains the same logs as those in $\sigma_{1}(R)$, the right hand bracket tends to a constant for small $R$ and all the $R$ dependence comes from the $\sigma^{\mathrm{LL}_{R}}(R)$ factor. Since $\sigma^{\mathrm{LL}_{R}}(R)$ tends to zero for $R \rightarrow 0$, eq. (3.3) satisfies the condition in eq. (3.2). The matching formula that we actually use is

$$
\sigma^{\mathrm{NLO}+\mathrm{LL}_{R}}=\left(\sigma_{0}+\sigma_{1}\left(R_{0}\right)\right) \times\left[\frac{\sigma^{\mathrm{LL}_{R}}(R)}{\sigma_{0}} \times\left(1+\frac{\sigma_{1}(R)-\sigma_{1}\left(R_{0}\right)-\sigma_{1}^{\mathrm{LL}_{R}}(R)}{\sigma_{0}}\right)\right],
$$

where $R_{0}$ is taken to be the same arbitrary radius of order 1 that appears in $\sigma^{\mathrm{LL}_{R}}(R)$ as defined in eq. (2.1). Compared to eq. (3.3), we have explicitly separated out a factor $\left(\sigma_{0}+\sigma_{1}\left(R_{0}\right)\right)$. As with eq. (3.3), at small- $R$ the entire $R$ dependence comes from the

\footnotetext{
${ }^{5}$ Once non-perturbative effects are accounted for, $\sigma^{\mathrm{LL}_{R}}(R=0)$ must coincide with the inclusive hadron spectrum.
} 


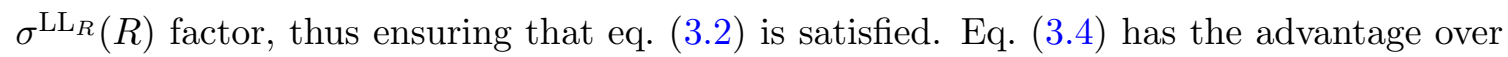
eq. (3.3) that matching will be simpler to extend to $\mathrm{NNLO}+\mathrm{LL}_{R}$, which is why we make it our default choice.

Eq. (3.4) has a simple physical interpretation: the left-hand factor is the cross section for producing a jet of radius $R_{0}$ and is effectively a stand-in for the normalisation of the (ill-defined) partonic scattering cross section, i.e. we equate partons with jets with radius $R_{0} \sim 1$. The right hand factor (in square brackets) then accounts for the effect of fragmentation on the cross section, including both the $\mathrm{LL}_{R}$ contribution and an exact NLO remainder for the difference between the cross sections at radii $R_{0}$ and $R$.

Even without a small- $R$ resummation, one can argue that the physical separation that is embodied in eq. (3.4) is one that should be applied to normal NLO calculations. This gives us the following alternative expression for the NLO cross section

$$
\sigma^{\mathrm{NLO}-\mathrm{mult.}}=\left(\sigma_{0}+\sigma_{1}\left(R_{0}\right)\right) \times\left(1+\frac{\sigma_{1}(R)-\sigma_{1}\left(R_{0}\right)}{\sigma_{0}}\right)
$$

i.e. the cross section for producing a small-radius jet should be thought of as the cross section for the initial partonic scattering, followed by the fragmentation of the parton to a jet. As in eq. (3.4), we introduce a radius $R_{0} \sim 1$ to give meaning to the concept of a "parton" beyond leading order. It is straightforward to see that eq. (3.5) differs from standard NLO only in terms of corrections at order $\alpha_{s}^{2}$ relative to LO.

\subsection{Unphysical cancellations in scale dependence}

Let us now return to the resummed matched prediction, eq. (3.4). The left and right-hand factors in that formula are shown separately in figure 3 . The left-hand factor, corresponding to an overall normalisation for hard partonic scattering, is shown in the left-hand plot (divided by the LO to ease visualisation), while the small- $R$ fragmentation (i.e. righthand) factor, which includes the resummation and matching contributions, is shown on the right. One sees that the two terms bring $K$-factors going in opposite directions. The overall normalisation has a $K$-factor that is larger than one and grows with $p_{t}$. Meanwhile the fragmentation effects generate a $K$-factor that is substantially below one for smaller $R$ values, with a relatively weak $p_{t}$ dependence.

The $p_{t}$ dependence of the two factors involves an interplay between two effects: on one hand, the fraction of gluons decreases at large $p_{t}$, as does $\alpha_{s}$; on the other hand the PDFs fall off more steeply at higher $p_{t}$, which enhances (positive) threshold logarithms in the normalisation factor and also increases the effect of small- $R$ logarithms in the fragmentation factor (i.e. reduces the fragmentation factor). We believe that the gentle increase of the fragmentation factor is due to the decrease in gluon fraction, partially counteracted by the increasing steepness of the PDFs. A similar cancellation is probably responsible for the flatness of the normalisation factor at low and moderate $p_{t}$ 's, with threshold logarithms induced by the PDFs' steepness driving the increase at the highest $p_{t}$ 's.

We note also that both factors in eq. (3.4) depend significantly on the choice of $R_{0}$, with two values being shown in figure $3, R_{0}=1$ (solid) and $R_{0}=1.5$ (dashed). However 

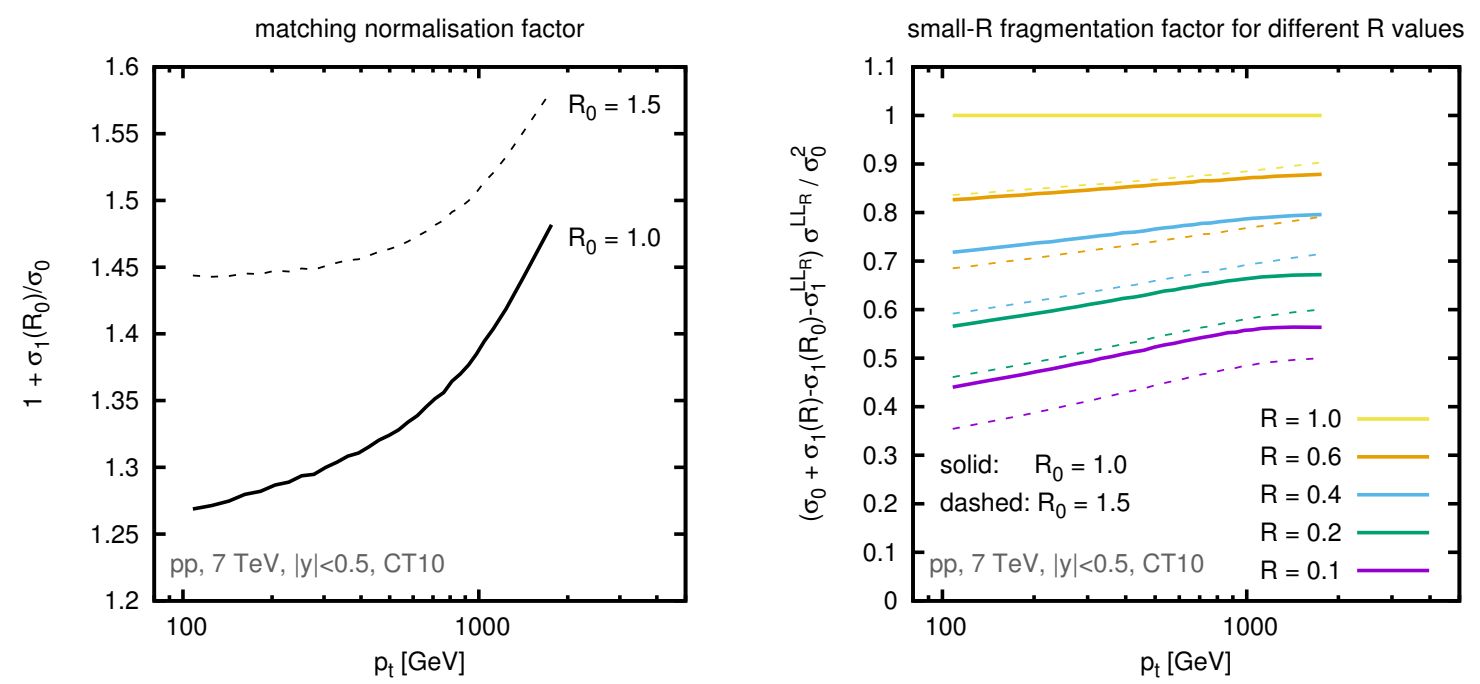

Figure 3. Left: size of the matching normalisation factor (left-hand factor of eq. (3.4), normalised to LO), shown v. $p_{t}$ for various $R$ values and two $R_{0}$ choices. Right: size of the matched small- $R$ fragmentation factor (right-hand factor of eq. (3.4); similar results are observed for the right-hand factor of eq. (3.5)). The results are shown for the scale choice $\mu_{R}=\mu_{F}=p_{t, \max }$, where $p_{t, \max }$ is the transverse momentum of the hardest jet in the event.

in the full results, eqs. (3.4) and (3.5), the $R_{0}$ dependence in cancels up to NLO, leaving a residual $R_{0}$ dependence that corresponds only to uncontrolled higher-order terms.

The partial cancellation between higher-order effects that takes place between the small- $R$ effects and the residual matching correction is somewhat reminiscent of the situation for jet vetoes in Higgs-boson production. There it has been argued that such a cancellation can be dangerous when it comes to estimating scale uncertainties. As a result, different schemes have been proposed to obtain a more reliable and conservative estimate, notably the Stewart-Tackmann [41] and jet-veto-efficiency [42] methods. Here we will take an approach that is similar in spirit to those suggestions (though somewhat closer to the jet-veto-efficiency method) and argue that for a reliable estimate of uncertainties, scale-dependence should be evaluated independently for the left and right-hand factors in eqs. (3.4) and (3.5) (and also in eq. (3.3)), and the resulting relative uncertainties on those two factors should be added in quadrature. We will always verify that the $R_{0}$ dependence (for just the central scale choice) is within our scale uncertainty band.

\section{$3.3 \quad \mathrm{NLO}+\mathrm{LL}_{R}$ matched results}

Figure 4 shows the inclusive jet cross section integrated from $100 \mathrm{GeV}$ to $1992 \mathrm{GeV}$ (the full range covered by the ATLAS data [27]), as a function of $R$, normalised to the leading order result. The left-hand plot shows the standard NLO result (light blue band), the "NLOmult." result of eq. (3.5) (green band) and the $\mathrm{NLO}+\mathrm{LL}_{R}$ matched result of eq. (3.4) (orange band). To illustrate the issue of cancellation of scale dependence discussed in section 3.2 , the scale uncertainty here has been obtained within a prescription in which the 

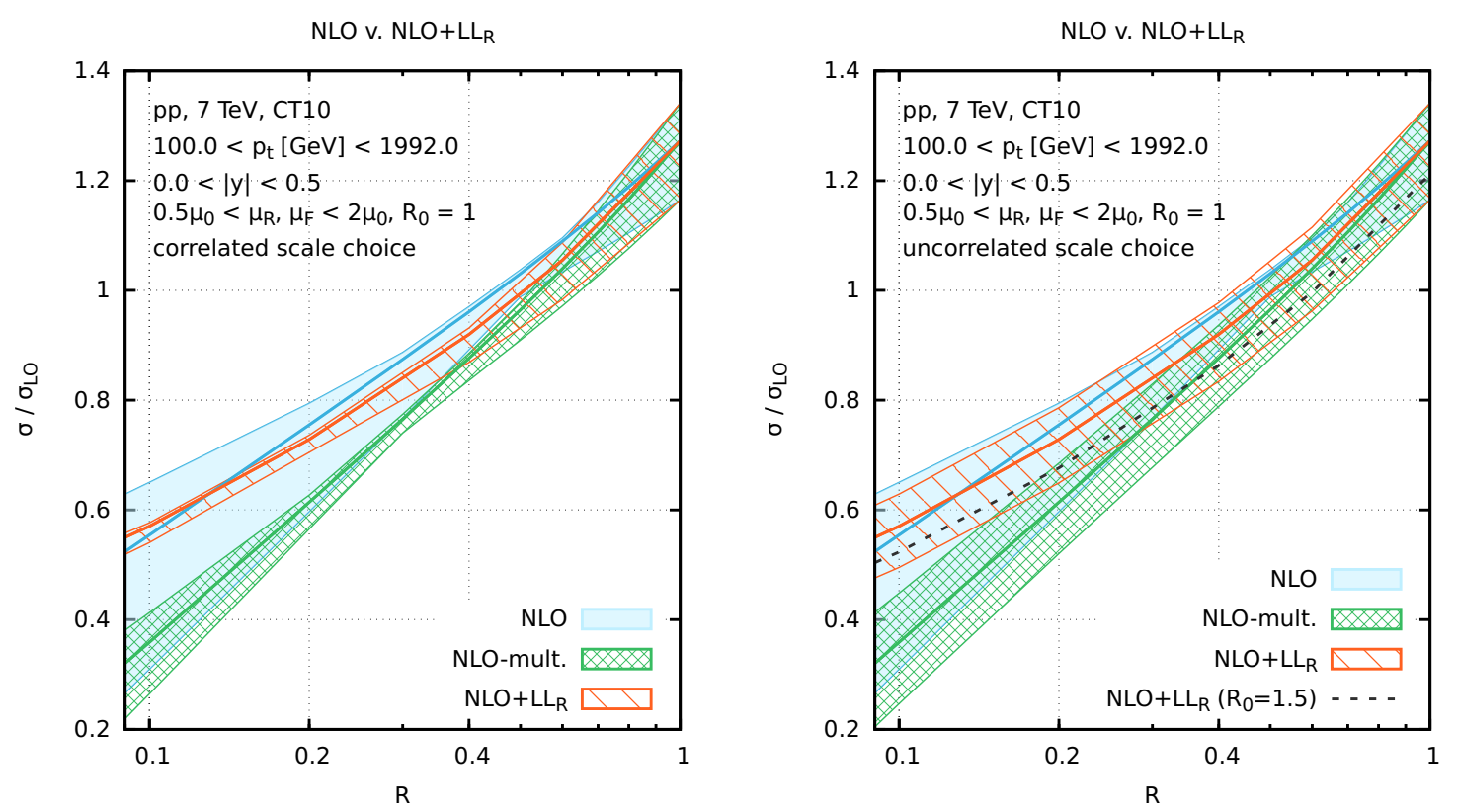

Figure 4. Inclusive jet cross section for $p_{t}>100 \mathrm{GeV}$, as a function of $R$, normalised to the $(R$ independent) leading-order result. Left: the standard NLO result, compared to the "NLO-mult." result of eq. (3.5) and the $\mathrm{NLO}+\mathrm{LL}_{R}$ matched result of eq. (3.4). The scale uncertainty here has been obtained within a prescription in which the scale is varied simultaneously in the left and righthand factors of eqs. (3.4) and (3.5) ("correlated scale choice"). Right: the same plot, but with the scale uncertainties determined separately the left and right-hand factors of eqs. (3.4) and (3.5), and then added in quadrature ("uncorrelated scale choice"). The plot also shows the NLO+LL $\mathrm{L}_{R}$ result for $R_{0}=1.5$ at our central scale choice.

scale is varied in a correlated way in the left and right-hand factors of eqs. (3.4) and (3.5). We adopt the standard convention of independent $\mu_{R}=\left\{\frac{1}{2}, 1,2\right\} \mu_{0}$ and $\mu_{F}=\left\{\frac{1}{2}, 1,2\right\} \mu_{0}$ variations around a central scale $\mu_{0}$, with the additional condition $\frac{1}{2} \leq \mu_{R} / \mu_{F} \leq 2$. Our choice for $\mu_{0}$ is discussed below.

One sees that in each of the 3 bands, there is an $R$ value for which the scale uncertainty comes close to vanishing, roughly $R=0.5$ for NLO, $R=0.3$ for "NLO-mult." and $R=0.1-0.2$ for $\mathrm{NLO}+\mathrm{LL}_{R}$. We believe that this near-vanishing is unphysical, an artefact of a cancellation in the scale dependence between small- $R$ and overall normalisation factors, as discussed in the previous paragraph. One clear sign that the scale dependence is unreasonably small is that the NLO-mult. and $\mathrm{NLO}+\mathrm{LL}_{R}$ bands differ by substantially more than their widths.

The right-hand plot of figure 4 instead shows uncertainty bands when one separately determines the scale variation uncertainty in the left-hand (normalisation) and the righthand (small- $R$ matching) factors and then adds those two uncertainties in quadrature ("uncorrelated scale choice"; note that the NLO band is unchanged). Now the uncertainties remain fairly uniform over the whole range of $R$ and if anything increase towards small $R$, as one might expect. This uncorrelated scale variation is the prescription that we will adopt for the rest of this article. 
Intriguingly, the $\mathrm{NLO}+\mathrm{LL}_{R}$ result is rather close to the plain NLO prediction. Given the large difference between the NLO and NLO-mult. results, this is, we believe, largely a coincidence: if one examines yet smaller $R$, one finds that the NLO and NLO+LL $R$ results separate from each other, with the NLO and NLO-mult. results going negative for sufficiently small $R$, while the $\mathrm{NLO}+\mathrm{LL}_{R}$ result maintains a physical behaviour.

Figure 4 (right) also shows the impact of increasing $R_{0}$ to 1.5 . One sees a $5-10 \%$ reduction in the cross section, however this reduction is within the uncertainty band that comes from the uncorrelated scale variation.

A comment is due concerning our choice of central scale, $\mu_{0}$. At NLO, for each event, we take $\mu_{0}$ to be the $p_{t}$ of the hardest jet in the event, $p_{t, \max }$. In NLO-like configurations, with at most 3 final-state partons, this hardest jet $p_{t}$ is independent of the jet radius and so we have a unique scale choice that applies to all jet radii. An alternative, widely used prescription is to use a separate scale for each jet, equal to that jet's $p_{t}$. We disfavour this alternative because it leads to a spurious additional $R$ dependence, induced by inconsistent scale choices in real and virtual terms. Further details are given in appendix B.

\section{Matching to NNLO}

\subsection{Matching prescription}

Given that full NNLO results for the inclusive cross section are likely to be available soon $[18,19]$, here we propose matching schemes for combining our small- $R$ resummed results with a full NNLO result. The direct analogue of eq. (3.4) is

$$
\begin{aligned}
& \sigma^{\mathrm{NNLO}+\mathrm{LL}_{R}}=\left(\sigma_{0}+\sigma_{1}\left(R_{0}\right)+\sigma_{2}\left(R_{0}\right)\right) \times \\
& \times\left[\frac{\sigma^{\mathrm{LL}_{R}}(R)}{\sigma_{0}} \times\left(1+\Delta_{1+2}\left(R, R_{0}\right)-\frac{\sigma_{1}^{\mathrm{LL}_{R}}(R)+\sigma_{2}^{\mathrm{LL}_{R}}(R)}{\sigma_{0}}\right.\right. \\
& \left.\left.\quad-\frac{\sigma_{1}^{\mathrm{LL}}(R)\left(\sigma_{1}(R)-\sigma_{1}^{\mathrm{LL}_{R}}(R)\right)}{\sigma_{0}^{2}}-\frac{\sigma_{1}\left(R_{0}\right)}{\sigma_{0}}\left(\Delta_{1}\left(R, R_{0}\right)-\frac{\sigma_{1}^{\mathrm{LL}_{R}}(R)}{\sigma_{0}}\right)\right)\right],
\end{aligned}
$$

where the functions $\Delta_{1}$ and $\Delta_{1+2}$ were defined in eq. (2.3) and (2.5) and we recall that $\sigma^{\mathrm{LL}_{R}}$ and its expansion are functions both of $R$ and $R_{0}$. As with our $\mathrm{NLO}+\mathrm{LL}_{R}$ formula, eq. (3.4), we have written eq. (4.1) in terms of two factors: an overall normalisation for producing $R_{0}$-jets, together with a matched fixed-order and resummed result for the correction coming from fragmentation of the $R_{0}$ jet into small- $R$ jets. One comment here is that in eq. (3.4) the matching part (big round brackets inside the square brackets) gave a finite result for $R \rightarrow 0$. The situation is different at NNLO because the $\mathrm{LL}_{R}$ resummation does not capture the $\alpha_{s}^{2} \ln 1 / R^{2}\left(\mathrm{NLL}_{R}\right)$ term that is present at fixed order and so the matching term has a residual $\alpha_{s}^{2} \ln 1 / R^{2}$ dependence. This means that for sufficiently small- $R$, eq. (4.1) can become unphysical. We have not seen any evidence of this occurring in practice, but one should keep in mind that for future work one might aim to resolve this in-principle problem either by incorporating $\mathrm{NLL}_{R}$ resummationor by choosing a different form of matching, for example one where the $\mathcal{O}\left(\alpha_{s}^{2}\right)$ parts of the matching correction are 
exponentiated, ensuring a positive-definite result. Note that with $\mathrm{NLL}_{R}$ resummation one could also use a formula analogous to eq. (3.3),

$$
\begin{aligned}
& \sigma^{\mathrm{NNLO}+\mathrm{NLL}_{R}, \text { mult.simple }}= \\
& \quad=\left(\sigma_{0}+\sigma_{1}+\sigma_{2}-\sigma_{1}^{\mathrm{NLL}_{R}}-\sigma_{2}^{\mathrm{NLL}_{R}}-\frac{\sigma_{1}^{\mathrm{NLL}_{R}}}{\sigma_{0}}\left(\sigma_{1}-\sigma_{1}^{\mathrm{NLL}_{R}}\right)\right) \times \frac{\sigma^{\mathrm{NLL}_{R}}}{\sigma_{0}},
\end{aligned}
$$

where each of the terms is evaluated at radius $R$.

As well as a matched result, it can be instructive to study a modification of the plain NNLO result, "NNLO-mult.", in analogy with eq. (3.5). This remains a fixed order result, but it factorises the production of large- $R_{0}$ jets from the fragmentation to small- $R$ jets,

$$
\sigma^{\mathrm{NNLO}-\mathrm{mult} .}=\left(\sigma_{0}+\sigma_{1}\left(R_{0}\right)+\sigma_{2}\left(R_{0}\right)\right) \times\left(1+\Delta_{1+2}\left(R, R_{0}\right)-\frac{\sigma_{1}\left(R_{0}\right)}{\sigma_{0}} \Delta_{1}\left(R, R_{0}\right)\right) .
$$

It differs from $\sigma^{\mathrm{NNLO}}$ only by terms beyond NNLO.

As in section 3.1, in eqs. (4.1)-(4.3) we advocate varying scales separately in the normalisation and fragmentation factors, and also studying the $R_{0}$ dependence of the final result.

\subsection{A stand-in for NNLO: $\mathrm{NNLO}_{R}$}

We have seen in section 2.2 that NNLO terms of the form $\alpha_{s}^{2} \ln 1 / R^{2}$ that are not accounted for in our $\mathrm{LL}_{R}$ calculation can be large. Insofar as they are known, they should however be included in phenomenological studies. This specific class of terms can be taken into account in the context of a stand-in for the full NNLO calculation which contains the exact NNLO $R$ dependence and that we refer to as $\mathrm{NNLO}_{R}$. It is constructed as follows:

$$
\sigma^{\mathrm{NNLO}_{R}}\left(R, R_{m}\right) \equiv \sigma_{0}+\sigma_{1}(R)+\left[\sigma_{2}(R)-\sigma_{2}\left(R_{m}\right)\right]
$$

which depends on an arbitrary angular scale $R_{m}$. Though neither $\sigma_{2}(R)$ nor $\sigma_{2}\left(R_{m}\right)$ can be fully determined currently, their difference can be obtained from the same NLO 3-jet calculation that was used to examine $\Delta_{1+2}\left(p_{t}, R, R_{\text {ref }}\right)$ in figure 2 (right).

Since the full NNLO result has the property

$$
\sigma^{\mathrm{NNLO}}(R)=\sigma^{\mathrm{NNLO}_{R}}\left(R, R_{m}\right)+\sigma_{2}\left(R_{m}\right),
$$

the use of $\sigma^{\mathrm{NNLO}_{R}}\left(R, R_{m}\right)$ instead of $\sigma^{\mathrm{NNLO}}(R)$ is equivalent to the assumption that $\sigma_{2}\left(R_{m}\right)$ vanishes. In practice we will take $R_{m}=1$, independently of $p_{t}$.

One point to be aware of is that $\sigma^{\mathrm{NNLO}_{R}}\left(R, R_{m}\right)$ and $\sigma^{\mathrm{NNLO}}(R)$ have parametrically different scale dependence. On one hand, the $\sigma_{2}(R)$ term in $\sigma^{\mathrm{NNLO}}(R)$ fully cancels the (relative) $\mathcal{O}\left(\alpha_{s}^{2}\right)$ scale variation that is left over from $\sigma_{0}$ and $\sigma_{1}$, leaving just $\mathcal{O}\left(\alpha_{s}^{3}\right)$ dependence. On the other, in $\sigma^{\mathrm{NNLO}_{R}}\left(R, R_{m}\right)$ the use of the $\sigma_{2}(R)-\sigma_{2}\left(R_{m}\right)$ means that some residual $\mathcal{O}\left(\alpha_{s}^{2}\right)$ dependence is left over. In particular, for $R=R_{m}$ the scale dependence is identical to that at NLO. Accordingly, when estimating higher-order uncertainties in 

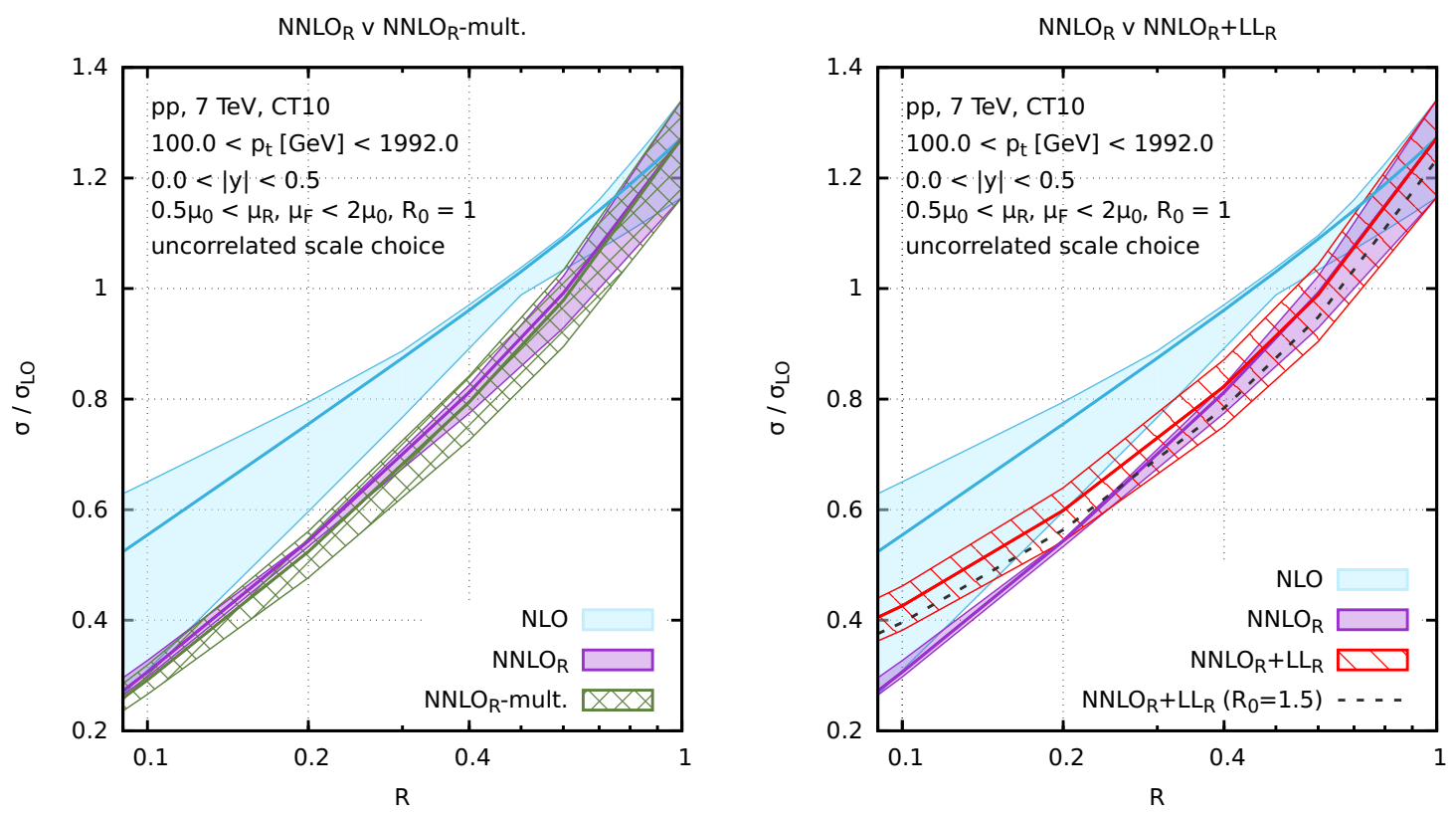

Figure 5. Left: comparison of the $\mathrm{NLO}, \mathrm{NNLO}_{R}$ and $\mathrm{NNLO}_{R}$-mult. results for the inclusive jet cross section for $p_{t}>100 \mathrm{GeV}$, as a function of $R$, normalised to the LO result. Right, corresponding comparison of NLO, $\mathrm{NNLO}_{R}$ and $\mathrm{NNLO}_{R}+\mathrm{LL}_{R}$ together with the central curve for $\mathrm{NNLO}_{R}+\mathrm{LL}_{R}$ when $R_{0}$ is increased to 1.5. In both plots, for the $\mathrm{NNLO}_{R}$-mult. and $\mathrm{NNLO}_{R}+\mathrm{LL}_{R}$ results the scaledependence has been evaluated separately in the normalisation and fragmentation contributions and added in quadrature to obtain the final uncertainty band.

studies that use $\mathrm{NNLO}_{R}$ results, we do not explicitly need to vary $R_{m}$, since the $\mathcal{O}\left(\alpha_{s}^{2}\right)$ uncertainty that it brings should already be accounted for in the scale variation. ${ }^{6}$

Our central scale choice for any given event will be $\mu_{0}=p_{t, \max }^{R=1}$, the transverse momentum of the hardest jet in the event as clustered with $R=1$. This is analogous to the choice of $p_{t, \text { max }}$ used at NLO, except that at NNLO one needs to explicitly specify $R$ since $p_{t, \text { max }}$ can depend on the jet clustering. The logic for taking $p_{t, \max }$ at a fixed jet radius of 1 , independently of the $R$ used in the clustering for the final jet spectrum, is that one obtains a unique scale for the event as a whole and avoids mixing scale-variation effects with $R$ dependence. Another potential choice that we did not investigate is to take the averaged $p_{t}$ of the two hardest jets. As long as the jets are obtained with a clustering radius $\sim 1$ such a choice is expected to be good at minimising the impact both of initial-state and final-state radiation, whereas our $p_{t, \text { max }}$ choice has some sensitivity to initial-state radiation.

\subsection{Results at $\mathrm{NNLO}_{R}$ and $\mathrm{NNLO}_{R}+\mathrm{LL}_{R}$}

Let us start by examining the $\mathrm{NNLO}_{R}$ result, shown versus $R$ as the purple band in figure 5 (left), together with the $\mathrm{NNLO}_{R}$-mult. results using eq. (4.3) and the NLO band. One sees that the $R$ dependence of the $\mathrm{NNLO}_{R}$ result is steeper than in the NLO result, especially for

\footnotetext{
${ }^{6}$ Despite this statement, one may wish to examine the robustness of conclusions with respect to different possibles values of $\sigma_{2}\left(R_{m}\right)$. This is the subject of section 4.4.
} 
$R \gtrsim 0.2$. This pattern is qualitatively in line with one's expectations from figure 2 (right) and will hold also for the full NNLO calculation, which differs from $\mathrm{NNLO}_{R}$ only by an $R$-independent (but $p_{t}$ and scale-dependent) additive constant. The point of intersection between the NLO and $\mathrm{NNLO}_{R}$ results, at $R=1$, is instead purely a consequence of our choice of $R_{m}=1$ in eq. (4.4). Thus at $R=1$, both the central value and scale dependence are by construction identical to those from the NLO calculation.

The left-hand plot of figure 5 also shows the $\mathrm{NNLO}_{R}$-mult. result. Relative to what we saw when comparing NLO and NLO-mult., the most striking difference here is the much better agreement between $\mathrm{NNLO}_{R}$ and $\mathrm{NNLO}_{R}$-mult., with the two generally coinciding to within a few percent. For $R \gtrsim 0.4$, this good agreement between different approaches carries through also to the comparison between $\mathrm{NNLO}_{R}$ and $\mathrm{NNLO}_{R}+\mathrm{LL}_{R}$. However, for yet smaller values of $R$, the $\mathrm{NNLO}_{R}+\mathrm{LL}_{R}$ result starts to be substantially above the $\mathrm{NNLO}_{R}$ and $\mathrm{NNLO}_{R}$-mult. ones. This is because the $\mathrm{NNLO}_{R}$ and $\mathrm{NNLO}_{R}$-mult. results have unresummed logarithms that, for very small- $R$, cause the cross section to go negative, whereas the resummation ensures that the cross section remains positive (modulo the potential issue with unresummed $\mathrm{NLL}_{R}$ terms that remain after matching).

Comparing the $\mathrm{NNLO}_{R}+\mathrm{LL}_{R}$ result to the $\mathrm{NLO}+\mathrm{LL}_{R}$ of figure 4 (right), one finds that the central value of the $\mathrm{NNLO}_{R}+\mathrm{LL}_{R}$ prediction starts to lie outside the $\mathrm{NLO}+\mathrm{LL}_{R}$ uncertainty band for $R \lesssim 0.5$. This highlights the importance of the NNLO corrections, and in particular of terms with subleading $\ln 1 / R^{2}$ enhancements. Finally, the dependence on the choice of $R_{0}$ is slightly reduced at $\mathrm{NNLO}_{R}+\mathrm{LL}_{R}$ compared to $\mathrm{NLO}+\mathrm{LL}_{R}$ and it remains within the scale-variation uncertainty band.

To help complete the picture, we also show results as a function of $R$ in a high- $p_{t}$ bin, $1530<p_{t}<1992 \mathrm{GeV}$ in figure 6 . Most of the qualitative observations that we discussed above remain true also for high $p_{t}$. The main difference relative to the $p_{t}>100 \mathrm{GeV}$ results is that scale uncertainty bands generally grow larger. This is perhaps due to threshold effects and might call for the inclusion of threshold resummation, see e.g. ref. [43] and references therein. Figures 7 and 8 show the jet spectrum as a function of $p_{t}$, normalised to the LO result, for $R=0.2$ and two rapidity bins. Again, the conclusions are similar.

All of the predictions shown here have been obtained with the choice $R_{m}=1$ in eq. (4.4), equivalent to the assumption that $\sigma_{2}\left(R_{m}=1\right)=0$ in eq. (4.5). For a discussion of how the predictions change if $\sigma_{2}\left(R_{m}=1\right)$ is non-zero, the reader is referred to section 4.4.

To conclude this section, our main observation is that $\mathrm{LL}_{R}$ and NNLO terms both have a significant impact on the $R$ dependence of the inclusive jet spectrum, with the inclusion of both appearing to be necessary in order to obtain reliable predictions for $R \lesssim 0.4$. In particular, if NNLO and NLO coincide for $R=1$, then for $R=0.4$ the NNLO results will be about $20 \%$ below the NLO ones. Going down to $R=0.2$, one sees that even with NNLO corrections resummation of small- $R$ logarithms is important, having a further $10 \%$ effect.

\subsection{Impact of finite two-loop corrections}

In our $\mathrm{NNLO}_{R}$-based predictions, we have all elements of the full NNLO correction except for those associated with 2-loop and squared 1-loop diagrams (and corresponding counterterms). 

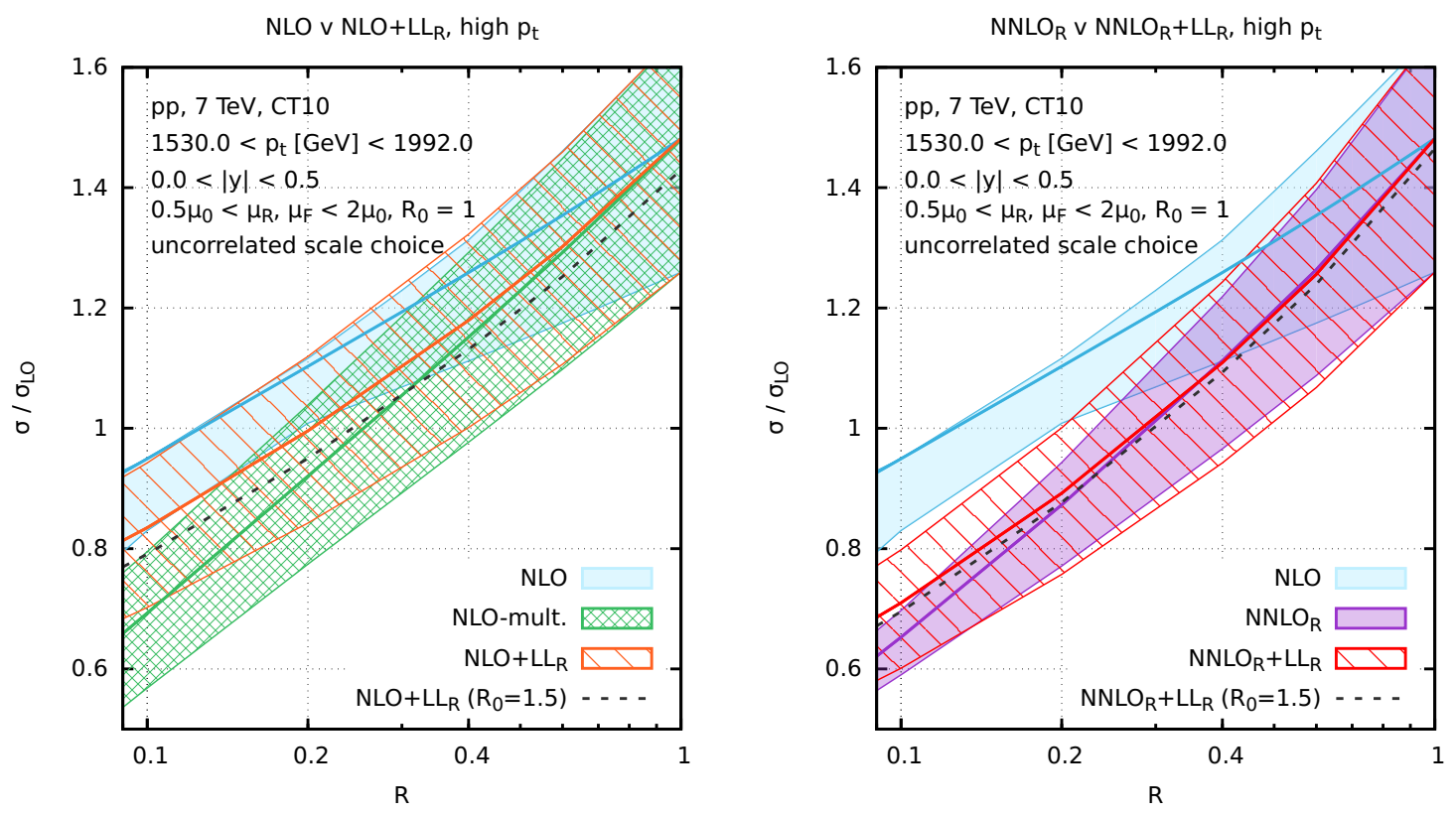

Figure 6. Same as figure 4 , but focusing only on the high $p_{t}$ bin. Both plots use an uncorrelated scale variation in the normalisation and fragmentation factors.
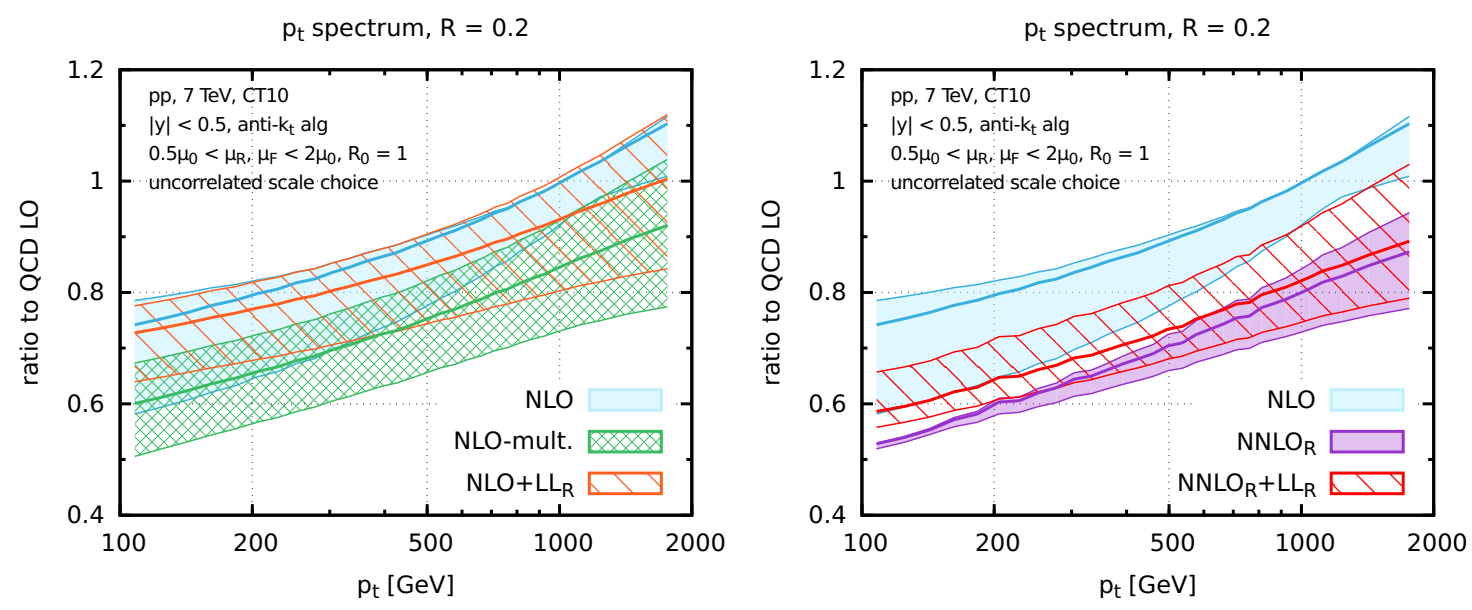

Figure 7. Left: the NLO inclusive jet spectrum as a function of $p_{t}$, normalised to LO, together with the "NLO-mult." result and the $\mathrm{NLO}+\mathrm{LL}_{R}$ matched results for $R=0.2$. The cross section is shown for the rapidity bin $|y|<0.5$. The bands give the scale uncertainty obtained using an uncorrelated scale choice. Right: analogous plots with the $\mathrm{NNLO}_{R}$ and $\mathrm{NNLO}_{R}+\mathrm{LL}_{R}$ predictions. 

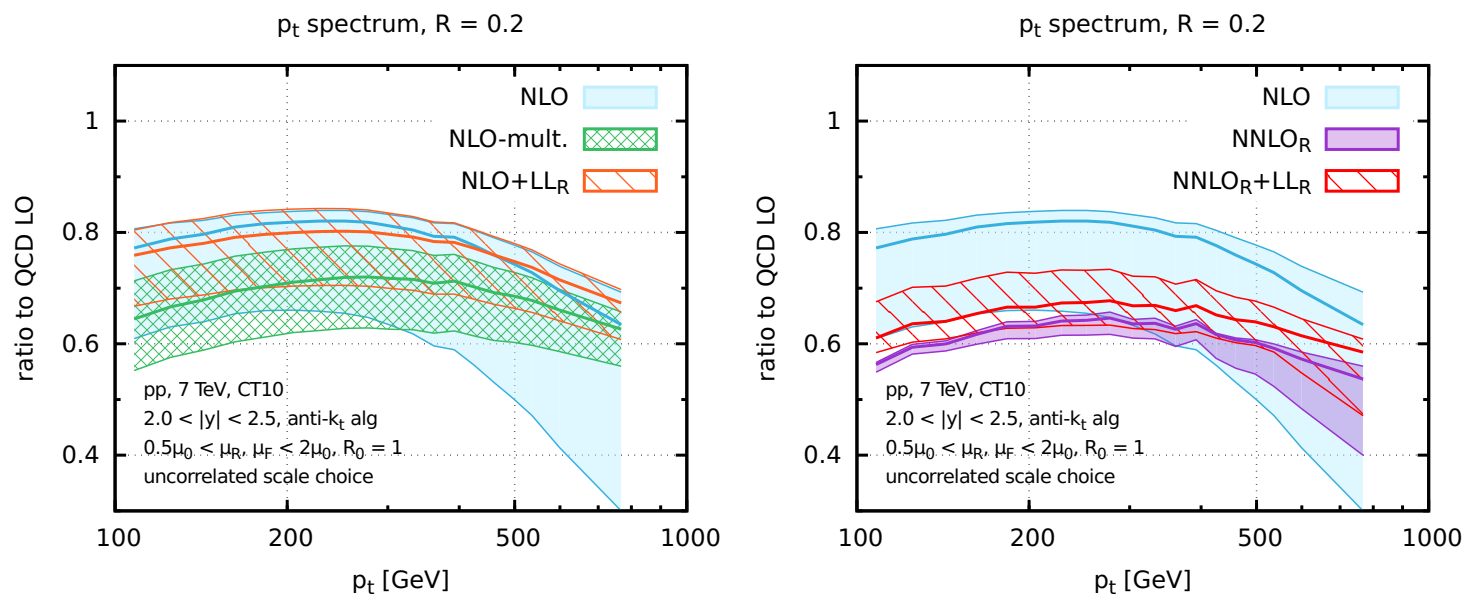

Figure 8. Same as figure 7 but showing the cross section in the rapidity bin $2<|y|<2.5$.

Here, we examine how our results depend on the size of those missing contributions. We introduce a factor $K$ that corresponds to the NNLO/NLO ratio for a jet radius of $R_{m}$ :

$$
\sigma^{\mathrm{NNLO}_{R, K}}\left(R_{m}\right)=K \times \sigma^{\mathrm{NLO}}\left(R_{m}\right) .
$$

For other values of the jet radius, we have

$$
\sigma^{\mathrm{NNLO}_{R, K}}(R)=\sigma_{0}\left[1+\frac{\sigma_{1}(R)}{\sigma_{0}}+\Delta_{2}\left(R, R_{m}\right)+(K-1) \times\left(1+\frac{\sigma_{1}\left(R_{m}\right)}{\sigma_{0}}\right)\right] .
$$

As before, we will take $R_{m}=1.0$. One could attempt to estimate $K$ from the partial NNLO calculation of ref. [18, 19], however given that this calculation is not yet complete, we prefer instead to leave $K$ as a free parameter and simply examine the impact of varying it.

In figure 9 , we show the impact of taking $K=1.10$, to be compared to figure 5 , which corresponds to $K=1$. As $K$ is increased, one sees that $\mathrm{NNLO}_{R, K}$ and $\mathrm{NNLO}_{R, K}+\mathrm{LL}_{R}$ start to agree over a wider range of $R$. This behaviour can be understood by observing there are two effects that cause $\mathrm{NNLO}_{R, K}$ and $\mathrm{NNLO}_{R, K}+\mathrm{LL}_{R}$ to differ: on one hand the small- $R$ resummation prevents the cross section from going negative at very small radii, raising the prediction in that region relative to $\mathrm{NNLO}_{R}$ and reducing the overall $R$ dependence. On the other hand, the normalisation (first) factor in eq. (4.1), which is larger than 1, multiplies the full NNLO $R$ dependence that is present in the fragmentation (second) factor, thus leading to a steeper $R$ dependence than in pure $\mathrm{NNLO}_{R, K}$. With $K=1$, the first effect appears to dominate. However as $K$ is increased, the second effect is enhanced and then the two effects cancel over a relatively broad range of $R$ values.

To put it another way, in the $\mathrm{NNLO}_{R, K}$ result the $K$ factor acts additively, shifting the cross section by the same amount independently of $R$. In the $\mathrm{NNLO}_{R, K}+\mathrm{LL}_{R}$ result, the $K$ factor acts multiplicatively, multiplying the cross section by a constant factor independently of $R$. By construction, the two always agree for $R=R_{0}=1$. With $K=1, \mathrm{NNLO}_{R, K}$ is below $\mathrm{NNLO}_{R, K}+\mathrm{LL}_{R}$ at small $R$, but the additive shift for $K>1$ brings about a larger 

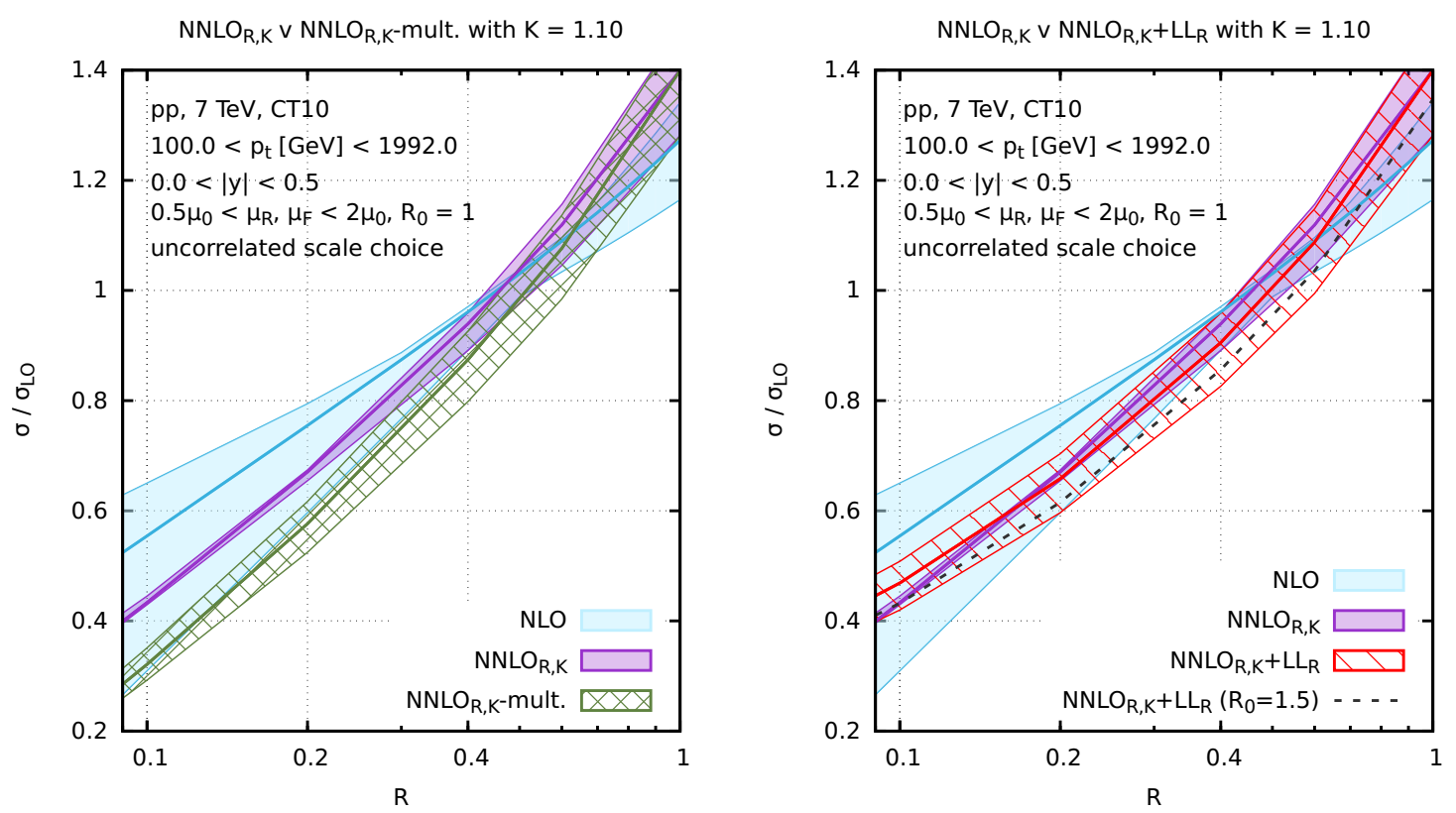

Figure 9. The same as figure 5, but applying a K-factor of 1.10 to the $\mathrm{NNLO}_{R}$ prediction, as an estimate of the potential impact of the full NNLO calculation.

increase of $\mathrm{NNLO}_{R, K}$ than the multiplicative factor for $\mathrm{NNLO}_{R, K}+\mathrm{LL}_{R}$, because $\sigma / \sigma\left(R_{0}\right)$ is smaller than one.

Another point to note is that while in figure 5 the $\mathrm{NNLO}_{R}$-mult. and $\mathrm{NNLO}_{R}$ results agreed over the full range of $R$, that is no longer the case with $K=1.1$ : this is because $\mathrm{NNLO}_{R}$-mult. acquires a multiplicative correction, as compared to the additive correction for $\mathrm{NNLO}_{R, K}$. Therefore one strong conclusion from our study is that independently of the size of the NNLO $K$-factor, plain fixed order calculations at NNLO are likely to be insufficient for $R \lesssim 0.4$.

\subsection{Comparison to POWHEG}

One widely used tool to study the inclusive jet spectrum is POWHEG's dijet implementation [44]. Insofar as parton showers should provide $\mathrm{LL}_{R}$ accuracy and POWHEG guarantees NLO accuracy, POWHEG together with a shower should provide NLO+LL $\mathrm{L}_{R}$ accuracy. It is therefore interesting to compare our results to those from the POWHEG BOX V2's dijet process (v3132), which are obtained here using a generation cut bornktmin of $50 \mathrm{GeV}$ and a suppression factor bornsuppfact of $500 \mathrm{GeV} .{ }^{7}$ We have used it with Pythia 8 (v8.186 with tune 4C [45]) for the parton shower, Pythia 6 (v6.428 the Perugia 2011 tune [46]) and with Herwig 6 (v6.521 with the AUET2-CTEQ6L1 tune [47]). We examine the results at parton level, with multiple-parton interaction (MPI) effects turned off. Since the Pythia 6 and Pythia 8 results are very similar we will show only the latter. In the case of Pythia 8,

\footnotetext{
${ }^{7}$ We also carried out a run with bornktmin of $25 \mathrm{GeV}$ and bornsuppfact of $300 \mathrm{GeV}$ and found results that are consistent with those shown here to within the statistical errors, which at low $p_{t}$ are of the order of $1 \%$.
} 

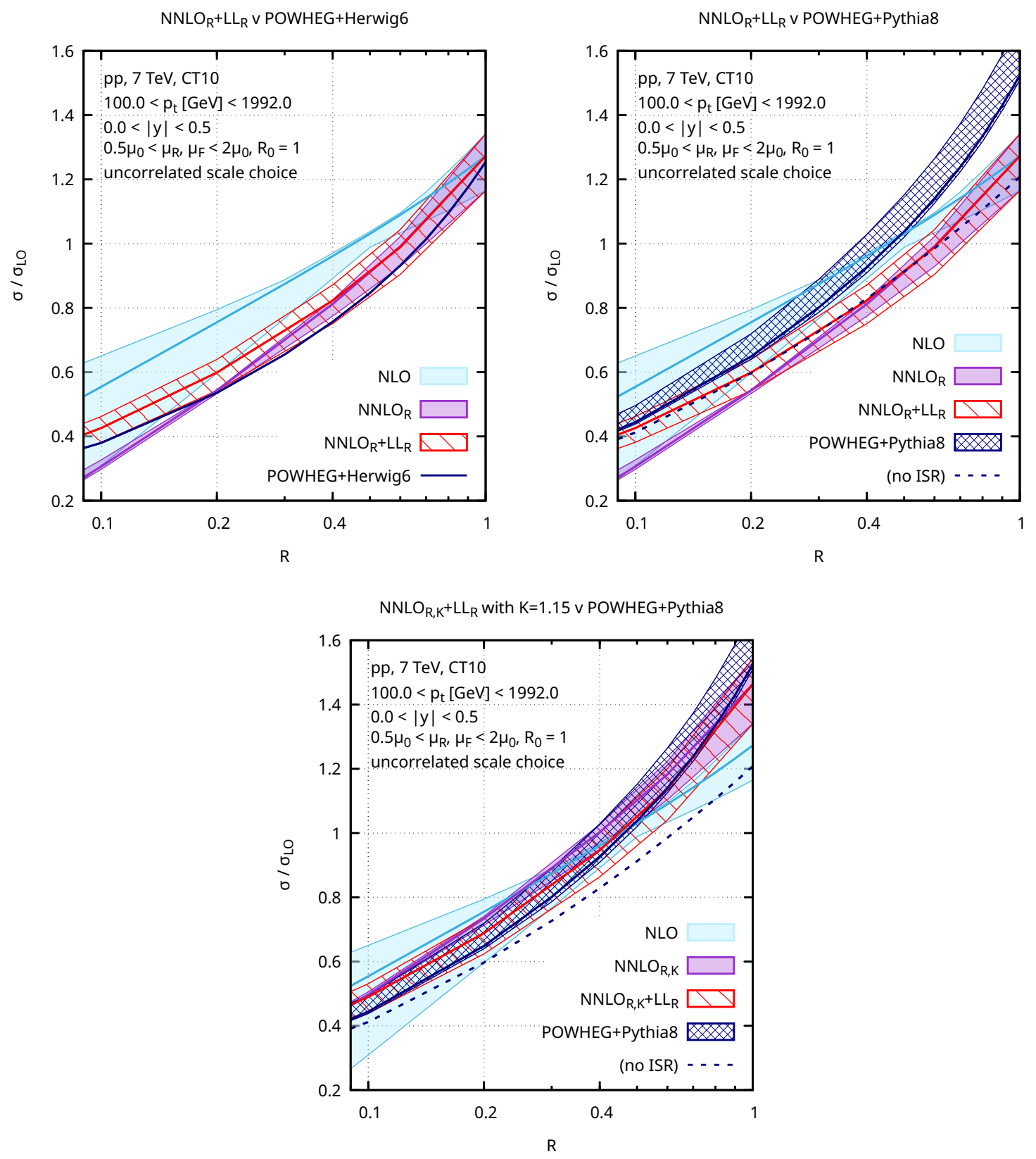

Figure 10. Top: comparison between the $\mathrm{NNLO}_{R}$-based results and POWHEG+Herwig 6 (left) and POWHEG+Pythia 8 (right), shown as a function of $R$, integrated over $p_{t}$ for $p_{t}>100 \mathrm{GeV}$. Bottom: comparison of POWHEG+Pythia 8 with $\mathrm{NNLO}_{R}$-based results, where the latter have an additional NNLO $K$-factor of 1.15 .

we include an uncertainty band from the variation of scales in the generation of the POWHEG events.

In figure 10, we show the $p_{t}$-integrated cross section as a function of $R$. The dark blue band (or line) shows the predictions obtained from POWHEG. In the top left-hand plot one sees a comparison with POWHEG+Herwig 6 , which agrees with the $\mathrm{NNLO}_{R}+\mathrm{LL}_{R}$ result 
to within the latter's uncertainty band, albeit with a slightly steeper $R$ dependence at large $R$ values. In the top right-hand plot, one sees a comparison with POWHEG+Pythia 8 . There is reasonable agreement for small radii, however the POWHEG+Pythia 8 prediction has much steeper $R$ dependence and is substantially above the $\mathrm{NNLO}_{R}+\mathrm{LL}_{R}$ result for $R=1$. Differences between Herwig and Pythia results with POWHEG have been observed before [48], though those are at hadron level, including underlying-event effects, which can introduce further sources of difference between generators.

One difference between the $\mathrm{NNLO}_{R}+\mathrm{LL}_{R}$ results and those from POWHEG with a showergenerator is an additional resummation of running scales and Sudakov effects for initialstate radiation (ISR). To illustrate the impact of ISR, the dark-blue dashed curve shows how the POWHEG+Pythia 8 prediction is modified if one switches off initial-state radiation (ISR) in the shower. Though not necessarily a legitimate thing to do (and the part of the ISR included in the POWHEG-generated emission has not been switched off), it is intriguing that this shows remarkably good agreement with the $\mathrm{NNLO}_{R}+\mathrm{LL}_{R}$ results over the full $R$ range. This might motivate a more detailed future study of the interplay between ISR and the jet spectrum. Note that, as shown in [44], nearly all the $R$ dependence of the POWHEG+partonshower result comes from the parton shower component. It is not so straightforward to examine Herwig with ISR turned off so we have not included this in our study.

Given the differences between POWHEG + Pythia 8 and our $\mathrm{NNLO}_{R}+\mathrm{LL}_{R}$ results, it is also of interest to examine what happens for $K \neq 1$. We can tune $K$ so as to produce reasonable agreement between $\mathrm{NNLO}_{R, K}+\mathrm{LL}_{R}$ and POWHEG+Pythia 8 for $R=1$ and this yields $K \simeq 1.15$, which we have used in the bottom-right plot. Then it turns out that both predictions agree within uncertainty bands not just at $R=1$, but over the full $R$ range. In this context it will be particularly interesting to see what effective value of $K$ comes out in the full NNLO calculation. Note that the patterns of agreement observed between different predictions depend also on $p_{t}$ and rapidity. For a more complete picture we refer the reader to our online tool [28].

\section{Hadronisation}

Before considering comparisons to data, it is important to examine also the impact of nonperturbative effects. There are two main effects: hadronisation, namely the effect of the transition from parton-level to hadron-level; and the underlying event (UE), generally associated with multiple interactions between partons in the colliding protons. Hadronisation is enhanced for small radii so we discuss it in some detail.

One way of understanding the effect of hadronisation and the underlying event is to observe that they bring about a shift in $p_{t}$. This can to some extent be calculated analytically and applied to the spectrum [20]. An alternative, more widespread approach is to use a Monte Carlo parton shower program to evaluate the ratio of hadron to parton level jet spectra and multiply the perturbative prediction by that ratio. One of the advantages of the analytical hadronisation approaches is that they can matched with the perturbative calculation, e.g. as originally proposed in ref. [49]. In contrast, a drawback of the Monte Carlo hadronisation estimates is that the definition of parton-level in a MC simulation is 

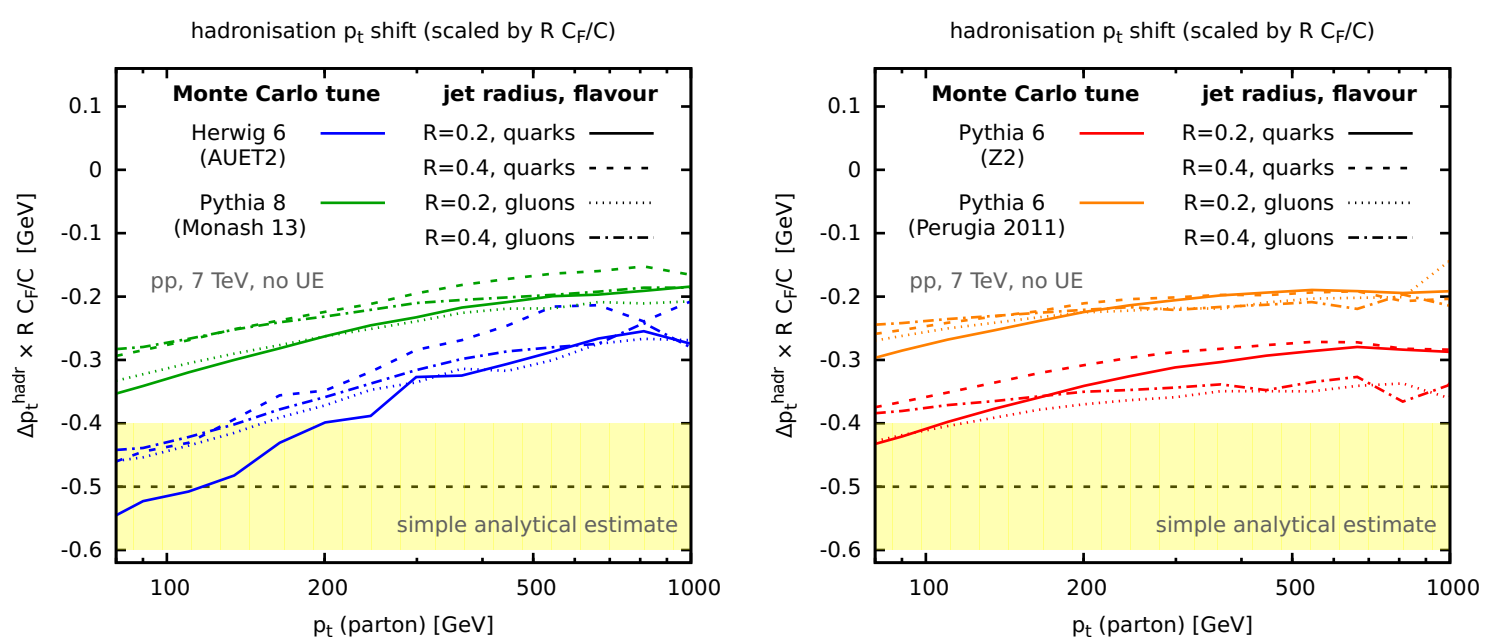

Figure 11. The average shift in jet $p_{t}$ induced by hadronisation in a range of Monte Carlo tunes, for $R=0.4$ and $R=0.2$ jets, both quark and gluon induced. The shift is shown as a function of jet $p_{t}$ and is rescaled by a factor $R C_{F} / C\left(C=C_{F}\right.$ or $\left.C_{A}\right)$ in order to test the scaling expected from eq. (5.1). The left-hand plot shows results from the AUET2 [47] tune of Herwig 6.521 [23, 24] and the Monash 13 tune [54] of Pythia 8.186 [22], while the right-hand plot shows results from the Z2 [55] and Perugia 2011 [46, 56] tunes of Pythia 6.428 [21]. The shifts have been obtained by clustering each Monte Carlo event at both parton and hadron level, matching the two hardest jets in the two levels and determining the difference in their $p_{t}$ 's. The simple analytical estimate of $0.5 \mathrm{GeV} \pm 20 \%$ is shown as a yellow band.

quite different from the definition of parton level that enters a perturbative calculation: in particular showers always include a transverse momentum cutoff at parton level, while perturbative calculations integrate transverse momenta down to zero.

To help guide our choice of method, we shall first compare the $p_{t}$ shift as determined in ref. [20] with what is found in modern Monte Carlo tunes. We first recall that the average shift should scale as $1 / R$ (see also refs. [50, 51]) for hadronisation and as $R^{2}$ for the underlying event (see also ref. [52]). For small- $R$ jets, hadronisation should therefore become a large effect, while the underlying event should vanish. By relating the hadronisation in jets to event-shape measurements in DIS and $e^{+} e^{-}$collisions in a dispersive-type model $[49,53]$, ref. [20] argued that the average $p_{t}$ shift should be roughly

$$
\left\langle\Delta p_{t}\right\rangle \simeq-\frac{C}{C_{F}}\left(\frac{1}{R}+\mathcal{O}(1)\right) \times 0.5 \mathrm{GeV},
$$

where $C$ is the colour factor of the parton initiating the jet, $C_{F}=\frac{4}{3}$ for a quark and $C_{A}=3$ for a gluon. Those expectations were borne out by Monte Carlo simulations at the time, with a remarkably small $\mathcal{O}(1)$ term. Eq. (5.1) translates to a $-6 \mathrm{GeV}$ shift for $R=0.2$ gluon-initiated jets. On a steeply falling spectrum, such a shift can modify the spectrum significantly.

Figure 11 shows the shift in $p_{t}$ in going from parton-level jets to hadron level jets, as a function of the jet $p_{t}$. Four modern Monte Carlo generator tunes are shown [21- 
$24,46,47,54,56]$, two in each plot. For each generator tune (corresponding to a given colour), there are four curves, corresponding to two values of $R, 0.2$ and 0.4 and both quark and gluon jets. The shifts have been rescaled by a factor $R C_{F} / C$. This means that if radius and colour-factor dependence in eq. (5.1) are exact, then all lines of a given colour will be superposed. This is not exactly the case, however lines of any given colour do tend to be quite close, giving reasonable confirmation of the expected trend of $C / R$ scaling.

A further expectation of eq. (5.1) is that the lines should cluster around $0.5 \mathrm{GeV}$ and be $p_{t}$ independent. This, however, is not the case. Firstly, there is almost a factor of two difference between different generators and tunes, with Pythia 6 Perugia 2011 and Pythia 8 Monash 2013 both having somewhat smaller than expected hadronisation corrections. Secondly there is a strong dependence of the shift on the initial jet $p_{t}$, with a variation of roughly a factor of two between $p_{t}=100 \mathrm{GeV}$ and $p_{t}=1 \mathrm{TeV}$. Such a $p_{t}$ dependence is not predicted within simple approaches to hadronisation such as refs. [20, 49, 50, 53]. It was not observed in ref. [20] because the Monte Carlo study there restricted its attention to a limited range of jet $p_{t}, 55-70 \mathrm{GeV}$. The event shape studies that provided support for the analytical hadronisation were also limited in the range of scales they probed, specifically, centre-of-mass energies in the range $40-200 \mathrm{GeV}$ (and comparable photon virtualities in DIS). Note, however, that scale dependence of the hadronisation has been observed at least once before, in a Monte Carlo study shown in figure 8 of ref. [57]: effects found there to be associated with hadron masses generated precisely the trend seen here in figure 11 . The $p_{t}$ dependence of those effects can be understood analytically, however we leave their detailed study in a hadron-collider context to future work. ${ }^{8}$ Experimental insight into the $p_{t}$ dependence of hadronisation might be possible by examining jet-shape measurements [59,60] over a range of $p_{t}$, however such a study is also beyond the scope of this work.

In addition to the issues of $p_{t}$ dependence, one further concern regarding the analytical approach is that it has limited predictive power for the fluctuations of the hadronisation corrections from jet to jet. Given that the jet spectrum falls steeply, these fluctuations can have a significant impact on the final normalisation of the jet spectrum. One might address this with an extension of our analytical approach to include shape functions, e.g. as discussed in ref. [61].

In light of the above discussion, for evaluating hadronisation effects here, we will resort to the standard approach of rescaling spectra by the ratio of hadron to parton levels derived from Monte Carlo simulations.

Figure 12 shows, as a function of $R$, the ratio of hadron-level without UE to parton-level (left) and the ratio of hadron level with UE to hadron level without UE (right), for a range of Monte Carlo tunes. The results are shown for $p_{t}>100 \mathrm{GeV}$ in the upper row and $p_{t}>1 \mathrm{TeV}$ in the lower row. A wide range of $R$ values is shown, extending well below experimentally accessible values. Beyond the tunes shown in figure 11, here we also include the UE-EE4 tune [62] of Herwig++ 2.71 [25, 26] and tune 4C [45] of Pythia 8.186 [22]. To investigate the issue of possible mismatch between our analytic parton-level calculations and partonlevel as defined in Monte Carlo simulations, we have considered a modification of Monte

\footnotetext{
${ }^{8}$ Hadron-mass effects have been discussed also in the context of ref. [58].
} 

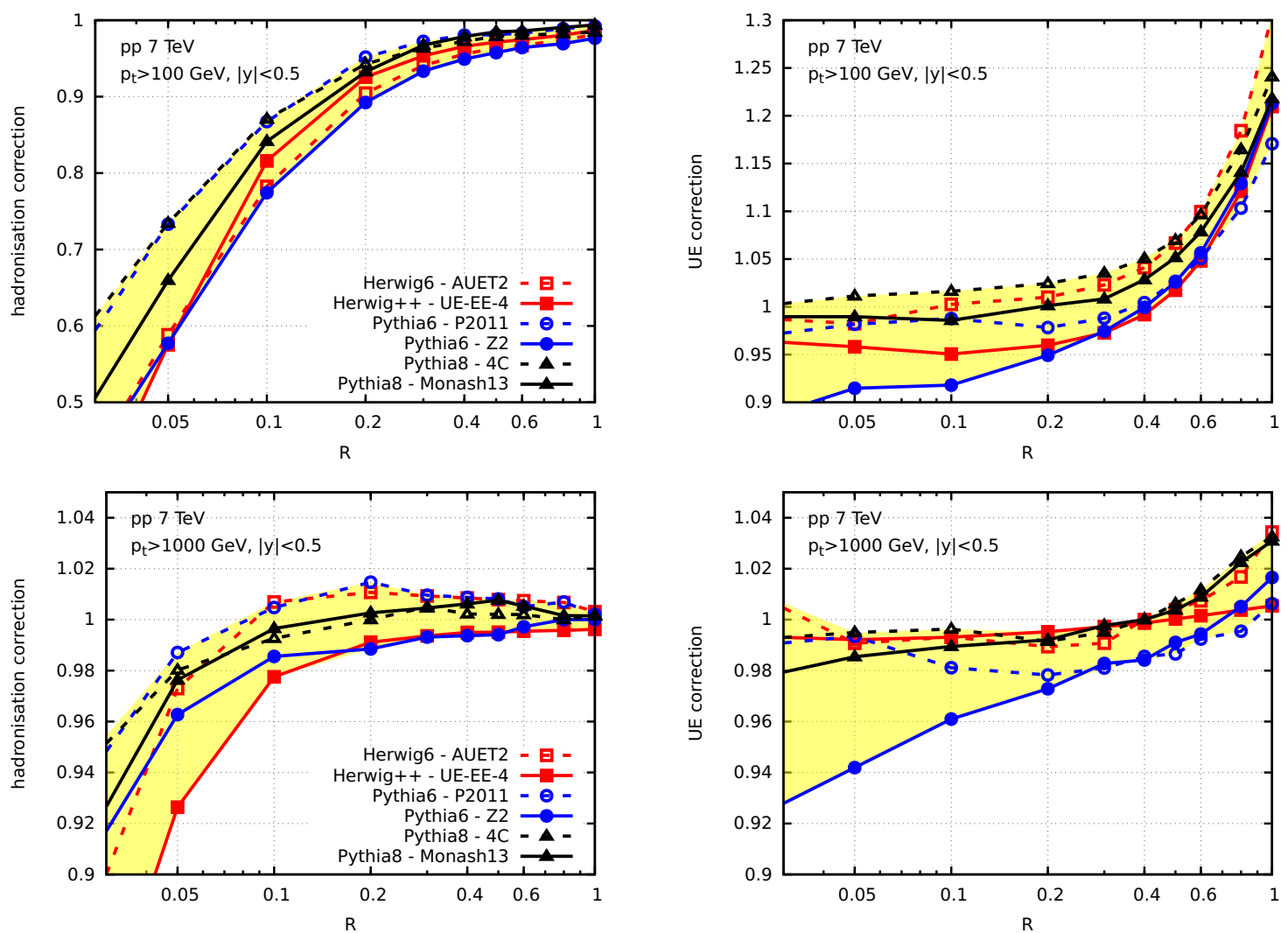

Figure 12. Hadronisation (left) and underlying event (right) multiplicative corrections to the jet spectrum, as a function of $R$ for pp collisions at $7 \mathrm{TeV}$. The top row shows results for $p_{t}>100 \mathrm{GeV}$ and $|y|<0.5$, while the bottom row is for $p_{t}>1 \mathrm{TeV}$. Six combinations of generator and tune are shown, and the yellow band corresponds to the envelope of the tunes.

Carlo parton level where the transverse momentum cutoff was taken to zero (an effective cutoff still remains, because of the use finite parton masses and $\Lambda_{\mathrm{QCD}}$ in the shower, however this method can arguably still give a rough estimate of the size of the effect one is dealing with). One finds that taking the cutoff to zero changes the parton-level spectrum by a few percent effect. As this is somewhat smaller than the differences that we will shortly observe between tunes, it seems that for the time being it may not be too unreasonable to neglect it.

While there is a substantial spread in results between the different tunes in figure 12, the observed behaviours are mostly as expected, with hadronisation reducing the jet spectrum, especially at the smallest $R$ values, while the UE increases it, especially at large $R$ values. The magnitude of these effects is strongly $p_{t}$ dependent, with (roughly) a factor of ten reduction at not-too-small $R$ values when going from $p_{t}>100 \mathrm{GeV}$ to $p_{t}>1 \mathrm{TeV}$. Such a scaling is consistent with a rough $1 /\left(R p_{t}\right)$ behaviour for hadronisation and $R^{2} / p_{t}$ behaviour for the UE (ignoring the slow changes in quark/gluon fraction and steepness of the spectrum as $p_{t}$ increases).

One surprising feature concerns the behaviour of the UE corrections at very small radii: firstly, in a number of the tunes the corrections tend to be smaller than 1, suggesting 
that the multi-parton interactions (MPI) that are responsible for the UE remove energy from the core of the jet. For $R$ values in the range $0.4-1$, the effect of MPI is instead to add energy to the jet, as expected. Secondly, this loss of energy from the jet is not particularly suppressed at high $p_{t}$. The most striking example is the Z2 tune where there can be corrections of up to $7 \%$ at $R=0.03$ (and even more at yet smaller $R$ values). The effect is somewhat reduced in the Z2-LEP tune, which has modified fragmentation parameters. One wonders if the mechanism for MPI generation might be inducing some form of factorisation breaking. A simple context in which to study this might be the high- $p_{t}$ inclusive hadron spectrum, where factorisation would imply that MPI should have no effect. While $k_{t}$-factorisation is believed to be broken for the inclusive hadron spectrum [63], we are not aware of definite statements concerning breaking of collinear factorisation.

\section{Comparisons to data}

Having formulated and studied the perturbative and non-perturbative contributions to the inclusive jet spectrum, we now consider comparisons with data. The purpose of this section is to highlight the relative sizes of different physical effects as compared to the precision of the data.

We will compare our predictions to the two datasets that have the smallest $R$ values: that from ALICE at centre-of-mass energy $\sqrt{s}=2.76 \mathrm{TeV}$ with $R=0.2$ and 0.4 [13] and that from ATLAS at $\sqrt{s}=7 \mathrm{TeV}$ with $R=0.4$ and 0.6 [27]. ${ }^{9}$

All our results are obtained with CT10 NLO PDFs. This is the case also for our LO and $\mathrm{NNLO}_{R}$ results. For the latter, since $\mathrm{NNLO}_{R}$ does not correspond to full NNLO, it is justifiable to use NLO PDFs. ${ }^{10}$ One should also be aware that most modern PDF sets include inclusive jet-data in their fit. Accordingly they may have a bias associated with the theory choice that was used in their determination. With an updated theoretical framework, such as that used here, the PDFs would conceivably change and a complete study would benefit from refitting the PDFs. That is beyond the scope of this work and anyway more appropriately done once full NNLO results become available. For completeness, we have nevertheless briefly examined the impact of changing PDFs in the context of a pure $\mathrm{LL}_{R}$ calculation, examining also CT10nnlo [66], CT14nlo, CT14nnlo [67], MSTW2008nlo [68], MMHT2014nlo, MMHT2014nnlo [69], NNPDF30_nlo_as_0118 and and NNPDF30_nnlo_as_0118 [70]. For $p_{t}$ 's below $500 \mathrm{GeV}$, most of these PDFs give results slightly above those from CT10, but by no more than $6 \%$, which is modest relative to other uncertainties and differences that we will see below.

All fixed-order results are obtained with version 4.1.3 of the NLOJet++ program [38]. Our central renormalisation and factorisation scale choice is $\mu_{0}=p_{t, \max }^{R=1}$, the transverse momentum of the hardest jet in the event as clustered with $R=1$. The envelope of independent variations of $\mu_{R}$ and $\mu_{F}$ by a factor of two (while maintaining $\frac{1}{2} \leq \mu_{R} / \mu_{F} \leq 2$ ) provides

\footnotetext{
${ }^{9}$ The CMS collaboration has also published inclusive jet spectrum results [64, 65], however the smallest $R$ considered there is slightly larger, $R=0.5$.

${ }^{10}$ In interpreting the plots, one may wish to keep in mind the potential impact of $K \neq 1$, which is illustrated explicitly in section 6.3. The plots use a $p_{t}$-independent NNLO $K$ factor, however the true $K$ factor would depend on $p_{t}$.
} 

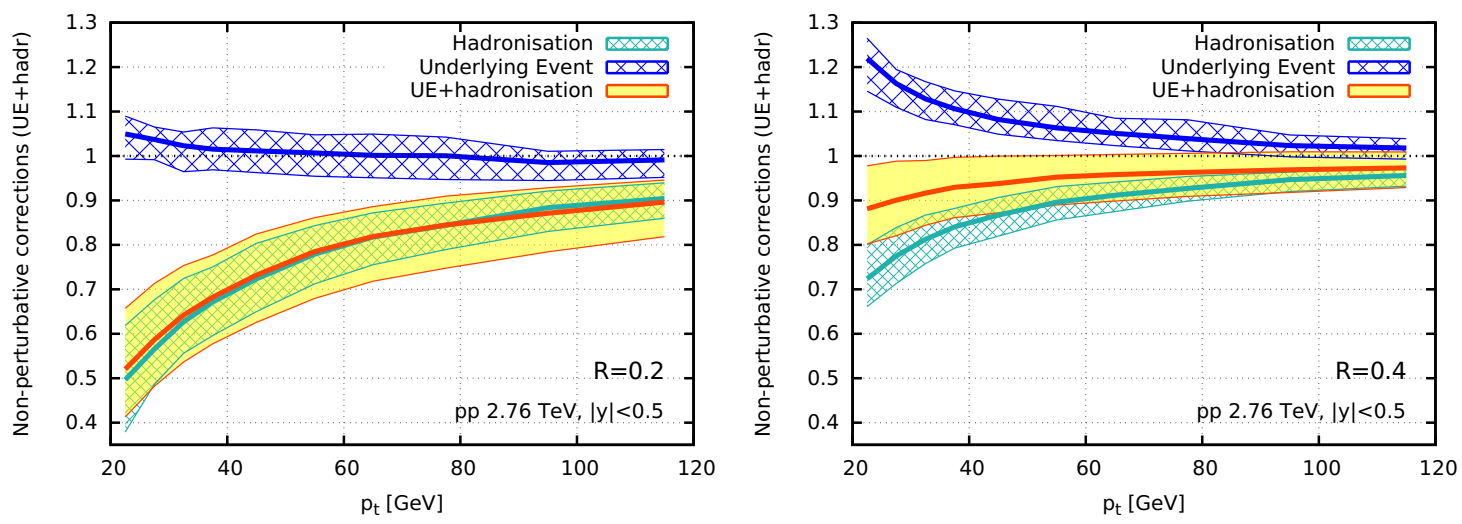

Figure 13. Non-perturbative corrections to the inclusive jet spectrum for the $p_{t}$ range, rapidity and centre-of-mass energy corresponding to the ALICE data [13] for $R=0.2$ (left) and $R=0.4$ (right). The results are shown separately for hadronisation, UE and the product of the two, and in each case include the average and envelope of the corrections from the six tunes discussed in section 5 .

the perturbative uncertainty estimate. In the case of NLO-mult. and $(\mathrm{N}) \mathrm{NLO}_{(R)}+\mathrm{LL}_{R}$ results, the scale variation is performed independently for the normalisation and fragmentation factors and the uncertainty from the two factors is then added in quadrature. As explained in section 3, this is intended to avoid spuriously small scale uncertainties associated with cancellations between different physical contributions.

Non-perturbative corrections are taken as the average of the parton-to-hadron Monte Carlo correction factors (including hadronisation and UE) as obtained with the six different tunes discussed in section 5 . The envelope of that set of six corrections provides our estimate of the uncertainty on the non-perturbative corrections, which is added in quadrature to the perturbative uncertainty.

In the case of the ATLAS data we will explore transverse momenta well above the electroweak (EW) scale, where EW corrections become substantial. The ATLAS collaboration accounted for these using the calculation of tree-level $\left(\mathcal{O}\left(\alpha_{s} \alpha_{\mathrm{EW}}\right)\right)$ and loop $\left(\mathcal{O}\left(\alpha_{s}^{2} \alpha_{\mathrm{EW}}\right)\right)$ EW effects from ref. [71]. Here, since we concentrate on QCD effects, when showing the data we divide it by the EW corrections quoted by ATLAS. ${ }^{11}$

\subsection{Comparison to ALICE data}

As a first application of small- $R$ resummation in comparisons to data, we look at the inclusive jet cross section in proton-proton collisions at $\sqrt{s}=2.76 \mathrm{TeV}$ reported by the ALICE collaboration [13]. The measurements are in the $|y|<0.5$ rapidity range, with jets obtained using the anti- $k_{t}$ algorithm with a boost-invariant $p_{t}$ recombination scheme, for radii $R=0.2$ and 0.4 .

\footnotetext{
${ }^{11}$ Those corrections don't account for real $W$ and $Z$ emission. The first estimate of real EW emission effects was given by Baur [72], but at the time only $14 \mathrm{TeV}$ collisions were envisaged. The real contributions for $7 \mathrm{TeV}$ collisions have been evaluated in ref. [73]. At high $p_{t}$ 's they grow to become up to $3-4 \%$, however in this region statistical and systematic uncertainties on the data are substantially larger and so we believe it is reasonable to neglect them.
} 

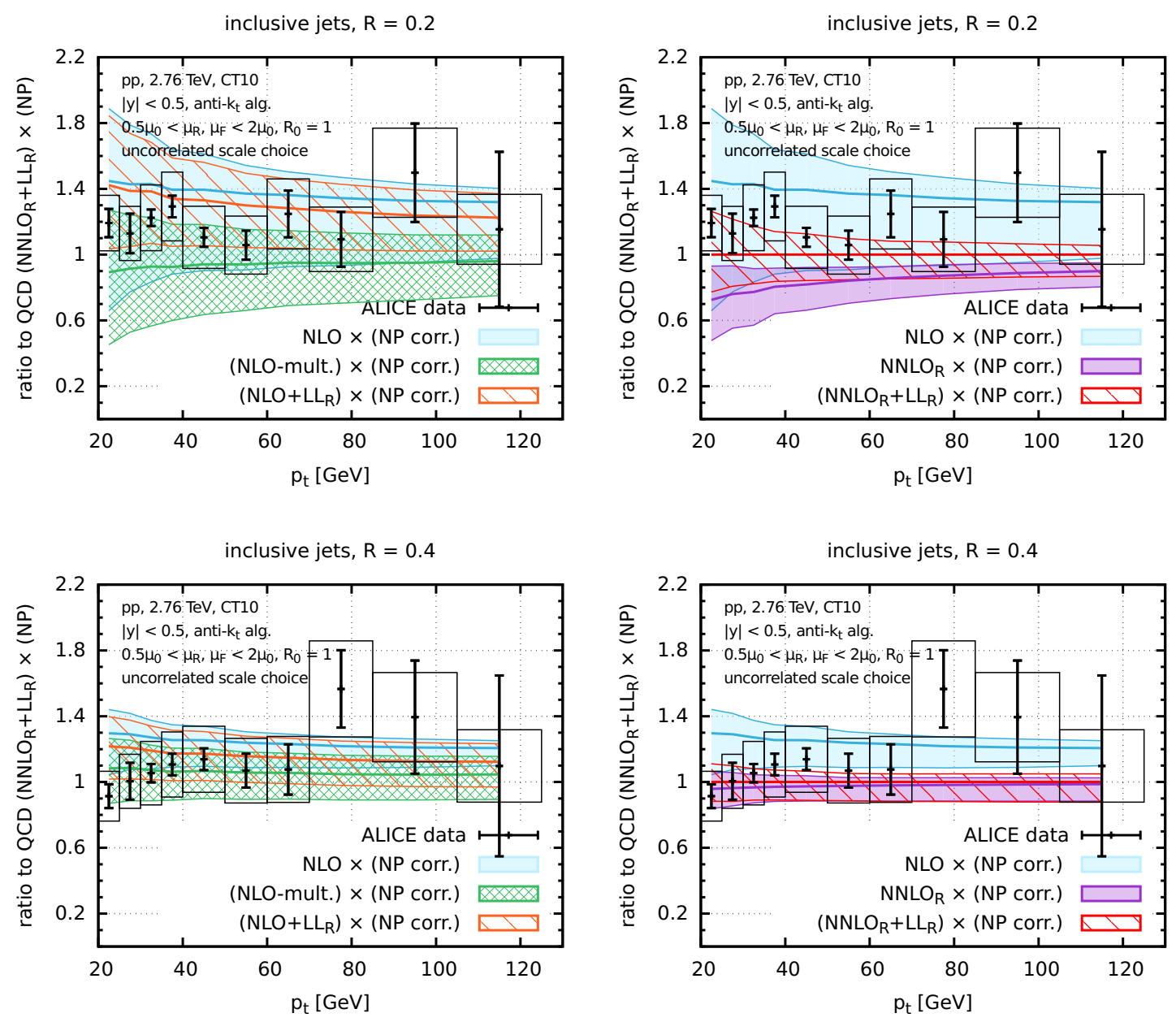

Figure 14. Comparison between a range of theoretical predictions for the inclusive jet spectrum and data from ALICE at $\sqrt{s}=2.76 \mathrm{TeV}$ [13]. The upper row is for $R=0.2$ and the lower one for $R=0.4$. The left-hand column shows NLO-based comparisons, while the right-hand one shows $\mathrm{NNLO}_{R}$-based comparisons. Rectangular boxes indicate the size of systematic uncertainties on the data points, while the errors bars correspond to the statistical uncertainties. Results are normalised to the central $\mathrm{NNLO}_{R}+\mathrm{LL}_{R}$ prediction (including non-perturbative corrections).

The non-perturbative corrections for hadronisation and underlying event are shown in figure 13. For $R=0.2$, non-perturbative corrections are largely dominated by hadronisation, with underlying event being a small effect, as expected for sufficiently small $R$. The net non-perturbative correction is about $-50 \%$ at the lowest $p_{t}$ of $20 \mathrm{GeV}$, while it decreases to about $-10 \%$ at $100 \mathrm{GeV}$. For $R=0.4$ there is a partial cancellation between hadronisation and UE, with a net impact of about $-10 \%$ percent at low $p_{t}$ and a $5-10 \%$ uncertainty.

The comparison of our full results to the ALICE data is given in figure 14, as a ratio to the $\mathrm{NNLO}_{R}+\mathrm{LL}_{R}$ theory prediction (including non-perturbative corrections). The top row shows the jet spectrum for $R=0.2$, while the lower row corresponds to $R=0.4$. The lefthand plots show NLO-based theory results. They all appear to be consistent with the data 
within their large uncertainties. The right-hand plots show $\mathrm{NNLO}_{R}$-based theory (with plain NLO retained to facilitate cross-comparisons). In general the $\mathrm{NNLO}_{R}+\mathrm{LL}_{R}$ results appear to provide the best match for the data, though they are slightly low. In particular, for $R=0.2$ where the differences between $\mathrm{NNLO}_{R}+\mathrm{LL}_{R}$ and $\mathrm{NNLO}_{R}$ are substantial, almost $30 \%$ at low $p_{t}$, there seems to be a preference for $\mathrm{NNLO}_{R}+\mathrm{LL}_{R}$. In contrast, at $R=0.4$ there is little difference between the two predictions though both are significantly more compatible with the data than is the plain NLO. In considering these statements, it is however important to keep several caveats in mind: the systematic uncertainties on the data and on the non-perturbative corrections (especially for $R=0.2$ ) are not negligible and a one- $\sigma$ shift could somewhat affect the conclusions. Furthermore, the currently unknown finite NNLO contribution (the difference between $\mathrm{NNLO}_{R}$ and full NNLO) may also have a relevant impact.

To further evaluate the compatibility of our results and the data we examine the ratio of the inclusive jet spectra at the two $R$ values, $\mathcal{R}\left(p_{t} ; R_{1}, R_{2}\right)=\sigma\left(p_{t} ; R_{1}\right) / \sigma\left(p_{t} ; R_{2}\right)$ with $R_{1}=0.2$ and $R_{2}=0.4$. This ratio is of interest because it allows us to directly study the $R$ dependence of the results and also because certain components of the uncertainties cancel in the ratio, in both the data and the theoretical prediction. In the experimental results, for example, the luminosity uncertainty cancels, as should part of the jet energy scale and resolution uncertainties. In the theoretical prediction, PDF uncertainties cancel. The ALICE collaboration's results [13] explicitly include a determination of the ratio.

Earlier studies that focused on the $R$ ratios [17] directly used the perturbative expansion for the cross-section ratio, rather than the ratio of perturbative predictions for the cross sections. That approach could be extended also to matched ratios, and one example of a $\mathrm{NNLO}+\mathrm{LL}_{R}$ matching formula for the ratio would be

$$
\begin{aligned}
\mathcal{R}^{\mathrm{NNLO}+\mathrm{LL}_{R}, \text { expand }}=\frac{\sigma^{\mathrm{LL}_{R}}\left(R_{1}\right)}{\sigma^{\mathrm{LL}}\left(R_{2}\right)} \times\left(1+\Delta_{1+2}\left(R_{1}, R_{2}\right)-\Delta_{1+2}\left(R_{1}, R_{2}\right) \frac{\sigma_{1}\left(R_{2}\right)}{\sigma_{0}}\right. \\
-\frac{\sigma_{1}^{\mathrm{LL}_{R}}\left(R_{1}\right)+\sigma_{2}^{\mathrm{LL}_{R}}\left(R_{1}\right)-\sigma_{1}^{\mathrm{LL}_{R}}\left(R_{2}\right)-\sigma_{2}^{\mathrm{LL}_{R}}\left(R_{2}\right)}{\sigma_{0}} \\
\left.+\left(\frac{\sigma_{1}^{\mathrm{LL}_{R}}\left(R_{1}\right)}{\sigma_{0}}-\Delta_{1}\left(R_{1}, R_{2}\right)\right) \frac{\sigma_{1}^{\mathrm{LL}_{R}}\left(R_{1}\right)-\sigma_{1}^{\mathrm{LL}_{R}}\left(R_{2}\right)}{\sigma_{0}}\right) .
\end{aligned}
$$

However, we prefer here to simply take the ratios of the relevant theory prediction (NLO, $\mathrm{NNLO}_{R}, \mathrm{NNLO}_{R}+\mathrm{LL} R$, etc.) at the two $R$ values, e.g.

$$
\mathcal{R}^{\mathrm{NNLO}+\mathrm{LL}_{R}}=\frac{\sigma^{\mathrm{NNLO}_{R}+\mathrm{LL}_{R}}\left(R_{1}\right)}{\sigma^{\mathrm{NNLO}_{R}+\mathrm{LL}_{R}\left(R_{2}\right)}} .
$$

This simple ratio has the same formal accuracy as (6.1) and fits better our primary goal, which is to predict inclusive jet cross sections and only examine their ratios for different $R$ values as a supplementary test. In the case of the results matched to $\mathrm{LL}_{R}$ resummation and of the (N)NLO-mult. results, the normalisation factor (with the cross section at radius $R_{0}$ ) cancels in the ratio, leaving only the fragmentation factor. For the $\mathrm{NNLO}_{R}$-mult. and $\mathrm{NNLO}_{R}+\mathrm{LL}_{R}$ results in particular, this means that any dependence on the unknown 

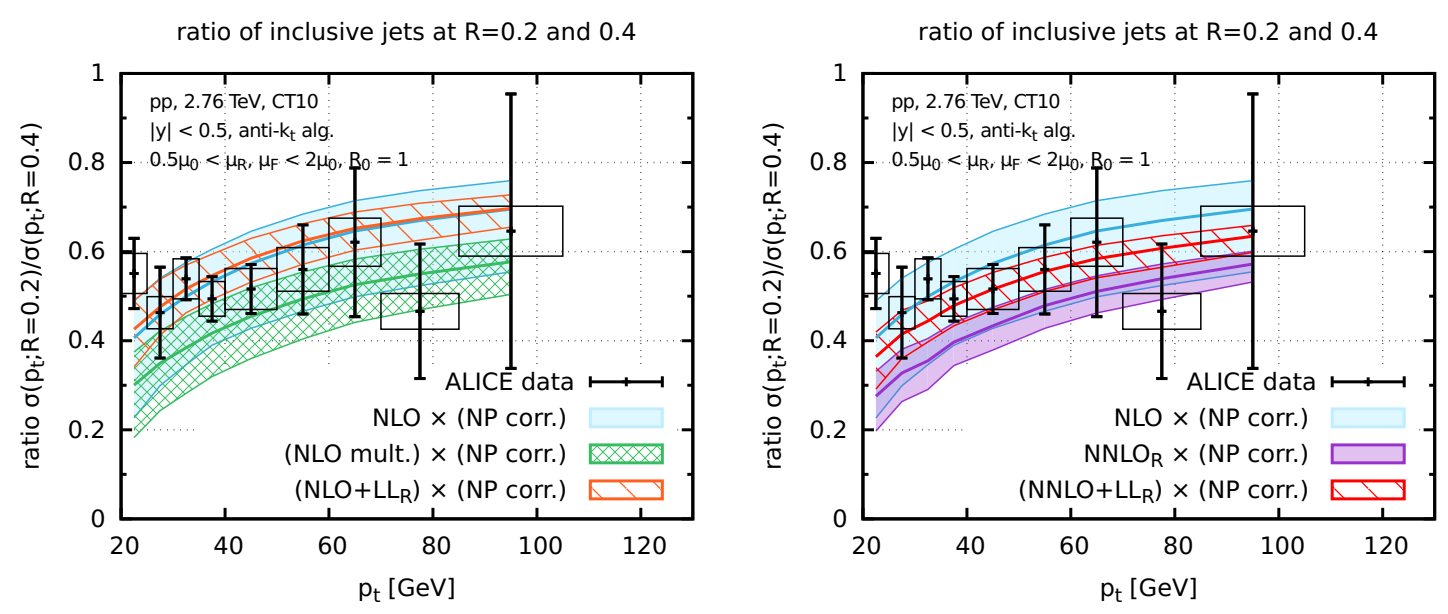

Figure 15. Comparison between a range of theoretical predictions for the inclusive jet crosssection ratio and data from ALICE at $\sqrt{s}=2.76 \mathrm{TeV}$ [13]. The left-hand column shows NLObased comparisons, while the right-hand one shows $\mathrm{NNLO}_{R}$-based comparisons. Rectangular boxes indicate the size of systematic uncertainties on the data points, while the errors bars correspond to the statistical uncertainties.

full NNLO $K$-factor (or, equivalently, the choice of $R_{m}$ in eq. (4.4)) is eliminated, and the prediction for the ratio is identical to that which would be obtained with the full NNLO result. Accordingly, we will drop the subscript ${ }_{R}$ label in these cases, i.e. writing $\mathcal{R}^{\mathrm{NNLO}+\mathrm{LL}_{R}}$ in eq. (6.2) rather than $\mathcal{R}^{\mathrm{NNLO}_{R}+\mathrm{LL}_{R}}$.

To estimate the perturbative theoretical uncertainties on the ratio, we take the envelope of the ratios as determined for our seven renormalisation and factorisation scale choices. In the case of $(\mathrm{N}) \mathrm{NLO}-$ mult. and $(\mathrm{N}) \mathrm{NLO}+\mathrm{LL}_{R}$ results, since the normalisation factor cancels, we only consider the component of the perturbative uncertainties associated with the fragmentation factor. We have verified that the effect of $R_{0}$ variation is contained within the scale-variation envelope. For the non-perturbative uncertainties, we take the envelope of the ratios of the corrections factors from different Monte Carlo tunes. The perturbative and non-perturbative uncertainties on the ratio are added in quadrature.

The comparison of the theory predictions with the measurements of the ALICE collaboration is presented in figure 15, at NLO accuracy on the left and at $\mathrm{NNLO}_{(R)}$-based accuracy on the right. At first sight, it appears that the data have a considerably flatter $p_{t}$ dependence than any of the theory predictions. The latter all grow noticeably with increasing $p_{t}$, a consequence mainly of the $p_{t}$ dependence of the non-perturbative correction factor, cf. figure 13. Nevertheless, on closer inspection one sees that if one ignores the left-most data point then the remaining data points are compatible with the predicted $p_{t}$ dependence. The overall agreement is then best with the NNLO $\mathrm{LL}_{R}$-based prediction. However, the sizes of the experimental uncertainties are such that it is difficult to draw firm conclusions.

We have also examined the impact of using eq. (6.1) instead of (6.2) and find that the difference is small, no more than $5 \%$. We have also examined the pure NNLO expansion of the ratio of cross sections, as used in ref. [17] and find that this too is quite similar to 

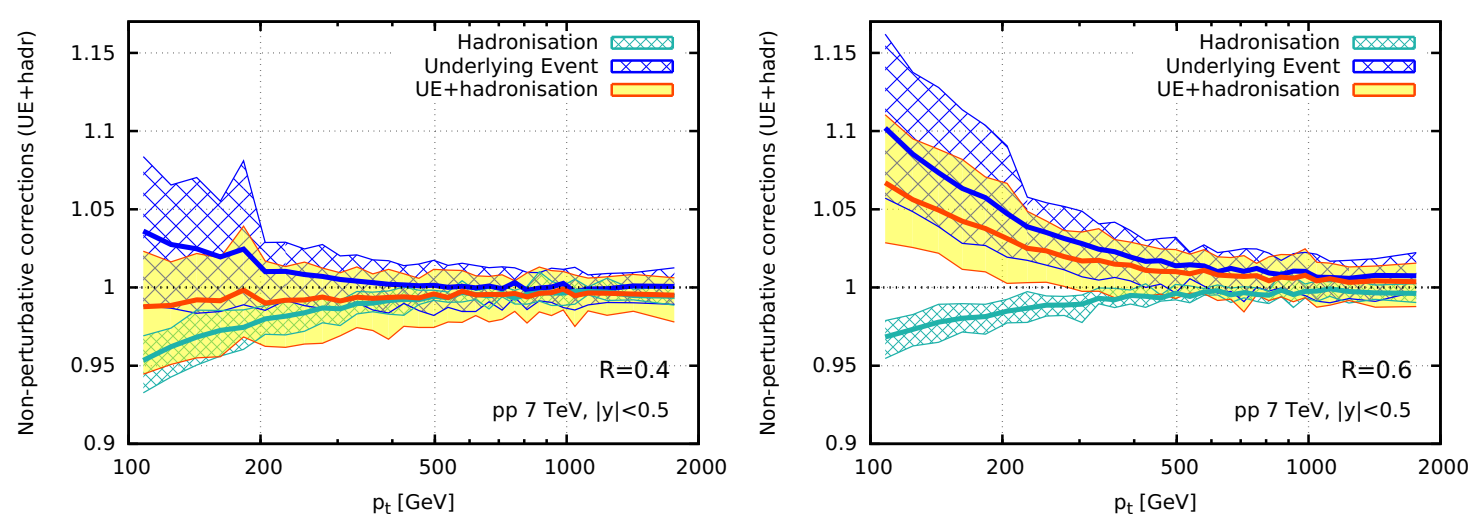

Figure 16. Non-perturbative corrections to the inclusive jet spectrum for the $p_{t}$ range, rapidity and centre-of-mass energy corresponding to the ATLAS data [27] for $R=0.4$ (left) and $R=0.6$ (right).

eq. (6.2), much more so than the direct ratio of NNLO results, $\sigma^{\mathrm{NNLO}_{R}}\left(R_{1}\right) / \sigma^{\mathrm{NNLO}_{R}}\left(R_{2}\right)$. Thus our finding that we obtain reasonable agreement between eq. (6.2) and the data is consistent with the observations of ref. [17], which were based on expanded NNLO ratios. ${ }^{12}$

\subsection{Comparison to ATLAS data}

Let us now turn to a comparison with the inclusive jet cross-sections reported by the ATLAS collaboration [27], obtained from $4.5 \mathrm{fb}^{-1}$ of proton-proton collisions at $\sqrt{s}=7 \mathrm{TeV}$. Jets are identified with the anti- $k_{t}$ algorithm, this time with a usual $E$-scheme, taking radii $R=0.4$ and 0.6. The measurements are doubly-differential, given as a function of jet $p_{t}$ and rapidity, and performed for $p_{t}>100 \mathrm{GeV}$ and $|y|<3$. Note that given the difference in centre-of-mass energy, the lower $p_{t}$ for the ATLAS data, $100 \mathrm{GeV}$, involves the same partonic $x$ range as $p_{t}=40 \mathrm{GeV}$ for the ALICE data.

The hadronisation and underlying event corrections applied are shown in figure 16. As in the case of the ALICE data, for $R=0.4$ these two classes of correction mostly cancel. When increasing the jet radius to $R=0.6$, the hadronisation corrections shrink, while the UE corrections increase and now dominate, leaving a net effect of up to $6-7 \%$ at the lowest $p_{t}$ 's.

Figures 17 and 18 show comparisons between data and theory for two rapidity bins, $|y|<0.5$ and $2.0<|y|<2.5$. At central rapidities the situation here contrasts somewhat with that for the ALICE data and in particular the inclusion of $\mathrm{NNLO}_{R}$ corrections worsens the agreement with data: over most of the $p_{t}$ range, the data points are about 15-20\% higher than than either $\mathrm{NNLO}_{R}$ or $\mathrm{NNLO}_{R}+\mathrm{LL}_{R}$ (which are close to each other, as expected for $R \gtrsim 0.4$ ). Nevertheless, one encouraging feature of the $\mathrm{NNLO}_{R}$-based predictions is that there is now a consistent picture when comparing $R=0.4$ and $R=0.6$, insofar as the ratio of data to $\mathrm{NNLO}_{R}$-theory is essentially independent of $R$. This is not

\footnotetext{
${ }^{12}$ Note, that ref. [17] used an analytical rather than Monte-Carlo based approach to estimating hadronisation corrections.
} 

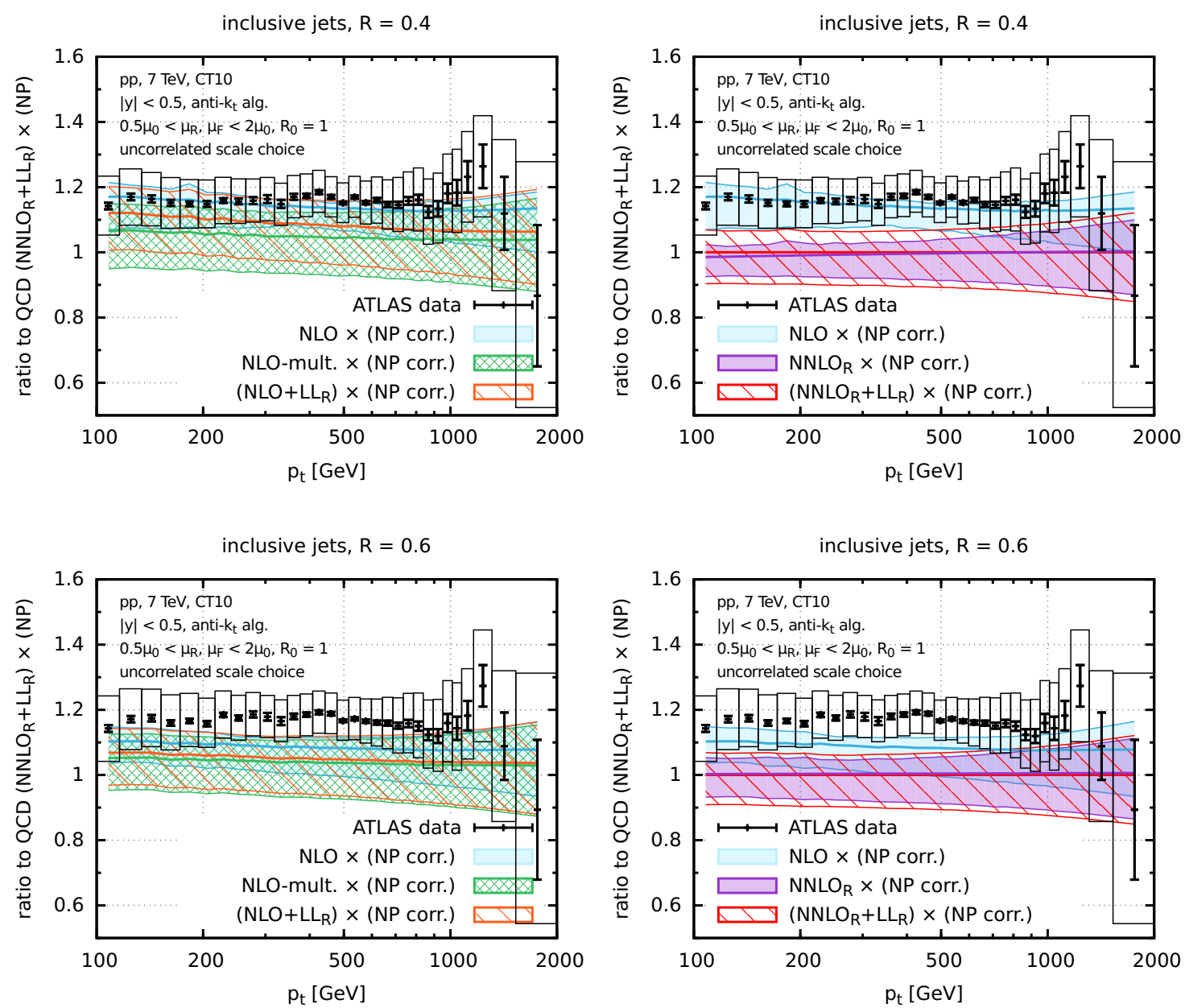

Figure 17. Comparison between a range of theoretical predictions for the inclusive jet spectrum and data from ATLAS at $\sqrt{s}=7 \mathrm{TeV}[27]$ in the rapidity bin $|y|<0.5$. The upper row is for $R=0.4$ and the lower one for $R=0.6$. The left-hand column shows NLO-based comparisons, while the right-hand one shows $\mathrm{NNLO}_{R}$-based comparisons. Rectangular boxes indicate the size of systematic uncertainties on the data points, while the errors bars correspond to the statistical uncertainties. Results are normalised to the central $\mathrm{NNLO}_{R}+\mathrm{LL}_{R}$ prediction (including non-perturbative corrections).

the case when comparing data and NLO predictions (cf. figure 5, which shows the steeper $R$ dependence of $\mathrm{NNLO}_{R}$-based results as compared to NLO). We return to the question of $R$ dependence in more detail below.

In the forward rapidity bin, over most of the $p_{t}$ range, the data instead favours the $\mathrm{NNLO}_{R}$-based predictions over NLO, while at high $p_{t}$ the data falls below all of the predictions. However the systematic uncertainties on the data are slightly larger than the difference with any of the theory predictions, making it difficult to draw any solid conclusions.

A significant positive 2-loop correction (cf. the discussion in sections 4.4, 4.5 and 6.3) would bring overall better agreement at central rapidities, but would worsen the agreement 
inclusive jets, $\mathrm{R}=0.4$

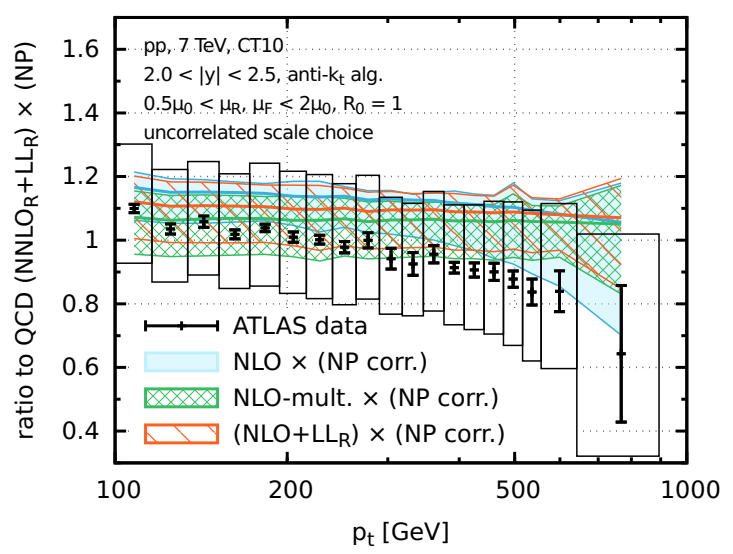

inclusive jets, $\mathrm{R}=0.6$

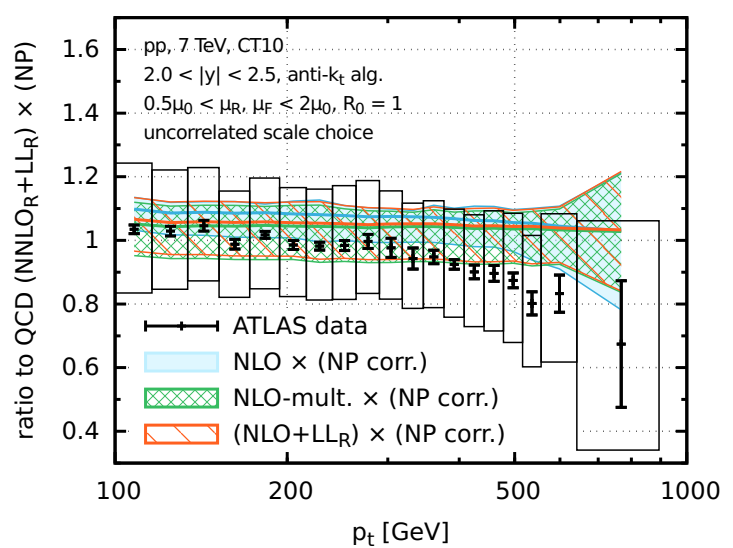

inclusive jets, $R=0.4$

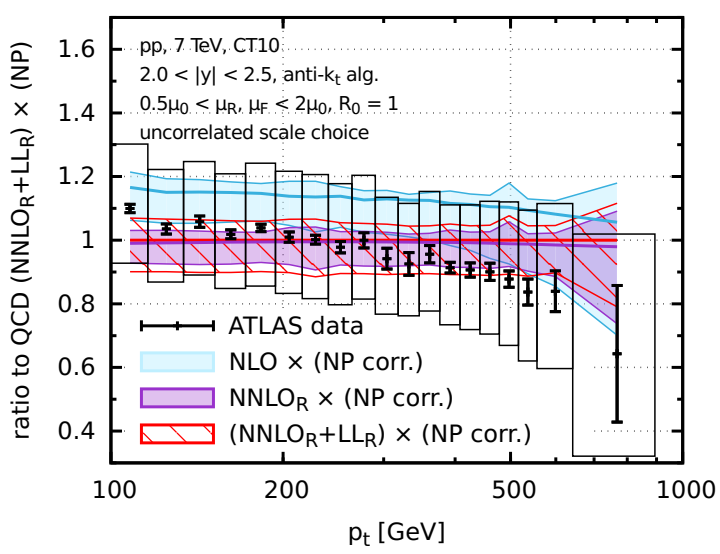

inclusive jets, $R=0.6$

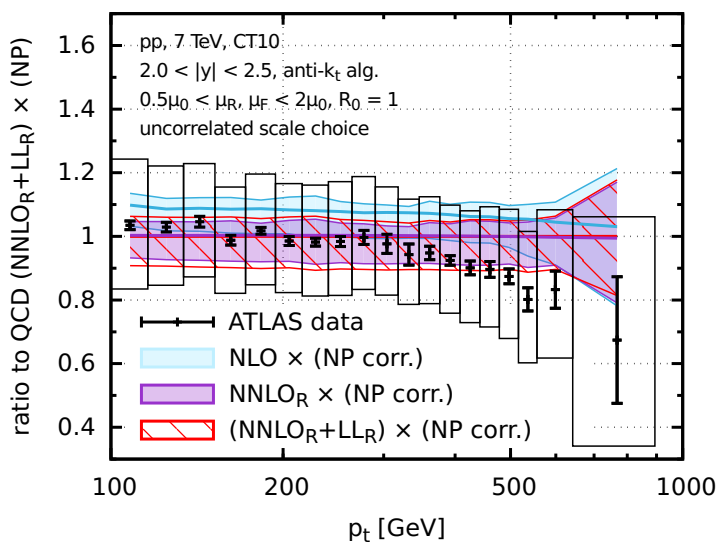

Figure 18. Analogue of figure 17, but for the rapidity bin $2<|y|<2.5$.

at forward rapidities. However, the finite 2-loop effects can be $p_{t}$ and rapidity dependent, making it difficult to draw any conclusions at this stage. Furthermore, one should keep in mind that adjustments in PDFs could affect different kinematic regions differently.

We close this section with an explicit comparison of the ratio of the jet spectra for the two different $R$ values. For the theoretical prediction, we proceed as discussed in the previous subsection, when we made a comparison with the ALICE data for such a ratio. We will not include EW effects, since in the ratio they appear to be at a level well below $1 \%$.

Concerning the experimental results, the central value of the ratio can be obtained directly from the ATLAS data at the two $R$ values. However the ATLAS collaboration has not provided information on the uncertainties in the ratio. It has provided information [74] to facilitate the determination of correlations between $p_{t}$ and rapidity bins, specifically 10000 Monte Carlo replicas of their data to aid in estimating statistical correlations, as well as a breakdown of systematic uncertainties into $\mathcal{O}(70)$ sources that are individually $100 \%$ correlated across bins and totally uncorrelated with each other. The information is 

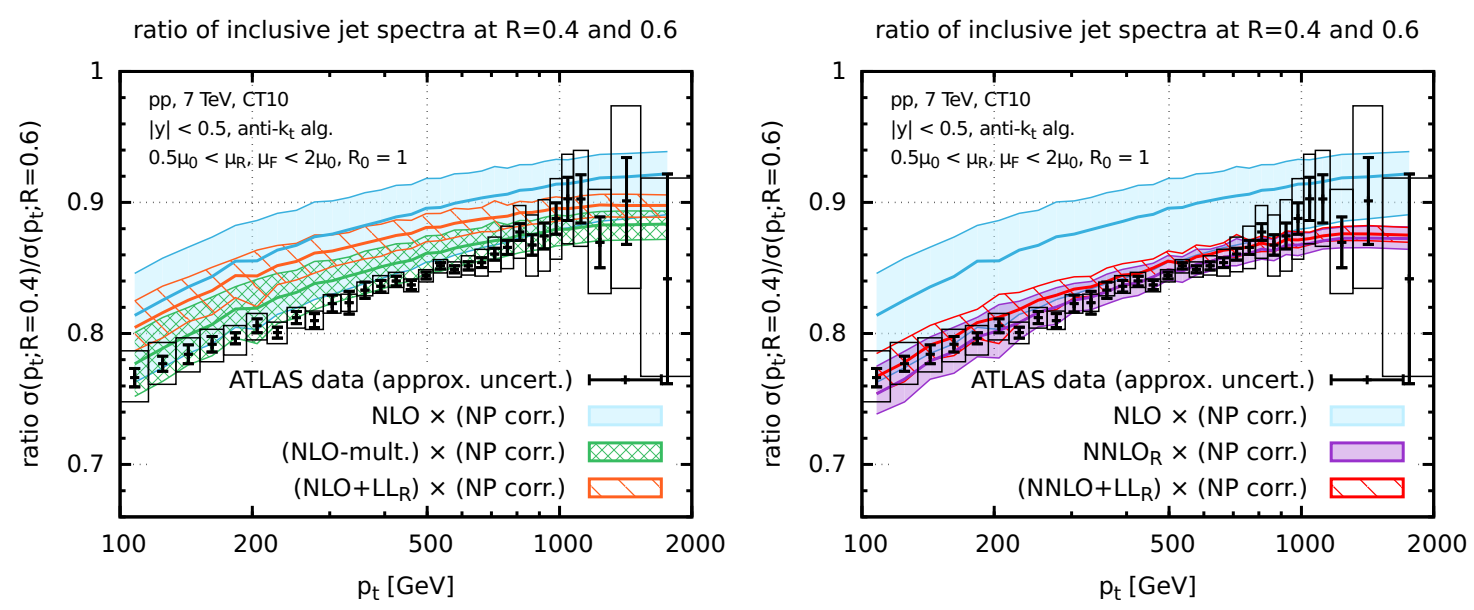

Figure 19. Comparison between a range of theoretical predictions for the inclusive jet crosssection ratio and data from ATLAS at $\sqrt{s}=7 \mathrm{TeV}$ [27]. The left-hand column shows NLO-based comparisons, while the right-hand one shows $\mathrm{NNLO}_{(R)}$-based comparisons. Rectangular boxes indicate our estimated systematic uncertainties on the data points, while the errors bars correspond to the statistical uncertainties. Note that these estimates are known to be incomplete, insofar as the information provided by the ATLAS collaboration on its results is not intended to be used for the determination of uncertainties on cross section ratios at different radii.

presented in a format such that, technically, it can also be used to estimate the uncertainties in the ratio of cross section for two $R$ values. However, we have been advised by the ATLAS collaboration that the degree of correlation between systematic uncertainties at different $R$ values is not well known. Accordingly, we label the uncertainties obtained in this way as "approx. uncert." to emphasise that we do not have full knowledge of the experimental uncertainties in the ratio and that they are potentially larger than our estimate.

Keeping in mind this caveat, we show in figure 19 a comparison between various theoretical predictions for the cross section ratio at $R=0.4$ relative to $R=0.6$, together with the experimental data. One sees overall very good agreement with both the $\mathrm{NNLO}_{R}$ and $\mathrm{NNLO}+\mathrm{LL}_{R}$-based results, and substantially worse accord with NLO-based predictions (albeit consistent with pure NLO and NLO-mult. within their larger uncertainties).

\subsection{Brief comparisons with an NNLO $K$-factor}

For completeness, here we show the comparisons between theoretical predictions and data change when we introduce a two-loop $K$-factor for $R=R_{m}$, as described in section 4.4. Figures 20, 21 and 22 are to be compared to their counterparts in sections 6.1 and 6.2 i.e. figures 14, 17 and 18. In most cases, the changes that one observes are largely as expected, with a corresponding trivial rescaling of the observed data-theory ratio. One exception is in the case of the $R=0.2$ comparison to ALICE data, figure 20 (left), where with $K=1.10$ one observes that the $\mathrm{NNLO}_{R, K}$ results are now in very close accord with the $\mathrm{NNLO}_{R, K}+\mathrm{LL}_{R}$ results. This is to be contrasted with the situation in figure 14. The 

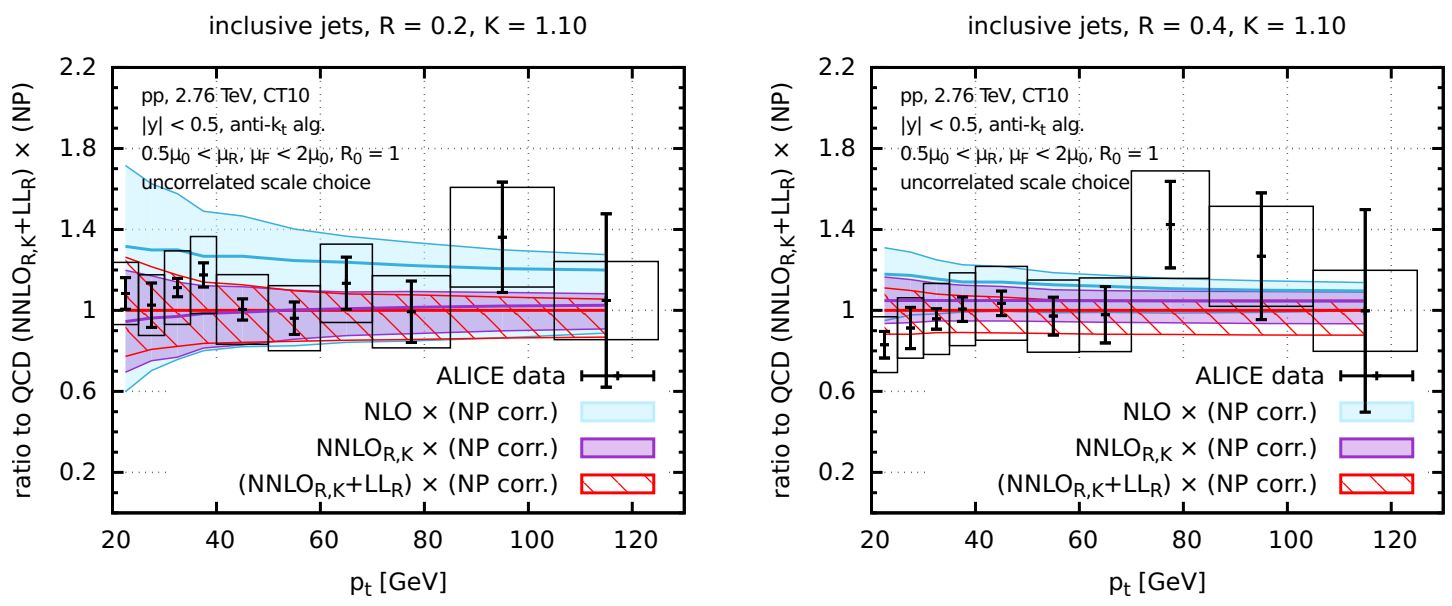

Figure 20. Comparison between theoretical predictions with a NNLO $R_{m}=1$ correction factor $K=1.10$ and data from ALICE at $\sqrt{s}=2.76 \mathrm{TeV}$ [13] at $R=0.2$ and $R=0.4$. Rectangular boxes indicate the size of systematic uncertainties on the data points, while the errors bars correspond to the statistical uncertainties. Results are normalised to the central $\mathrm{NNLO}_{R, K}+\mathrm{LL}_{R}$ prediction (including non-perturbative corrections).

difference is due to the fact that the $K$ factor acts additively on the $\mathrm{NNLO}_{R, K}$ result, but multiplicatively on the $\mathrm{NNLO}_{R, K}+\mathrm{LL}_{R}$ result, as discussed already in section 4.4.

Note that for the ATLAS comparison, while a $K$-factor of $K=1.10$ improves agreement with the data at central rapidities, it appears to worsen it somewhat at high rapidities, as can be seen in figure 22. One should, however, keep in mind that the true $K$-factor will depend both on rapidity and $p_{t}$, and also that modifications associated with changes in PDFs can affect forward and central rapidities differently.

\section{Conclusion}

In this paper we have used the limit of small-radius jets to explore a variety of features of the most basic of jet observables, the inclusive jet spectrum.

A first observation, in section 2, was that the small- $R$ approximation starts to reproduce fixed-order $R$ dependence quite well already for $R$ just below 1 , giving us confidence in the usefulness of that approximation for phenomenologically relevant $R$ values.

In seeking to combine small- $R$ resummation with NLO predictions, in section 3 , it was natural to write the cross section as a product of two terms: an overall normalisation for elementary partonic scattering, together with a factor accounting for fragmentation of those partons into small- $R$ jets. Such a separation can be performed also at fixed order. There appear to be spurious cancellations between higher-order contributions for the two factors and this led us to propose that one should estimate their scale uncertainties independently and then add them in quadrature. This procedure has similarities with methods used for jet vetoes in Higgs physics [41, 42]. 
inclusive jets, $\mathrm{R}=0.4, \mathrm{~K}=1.10$

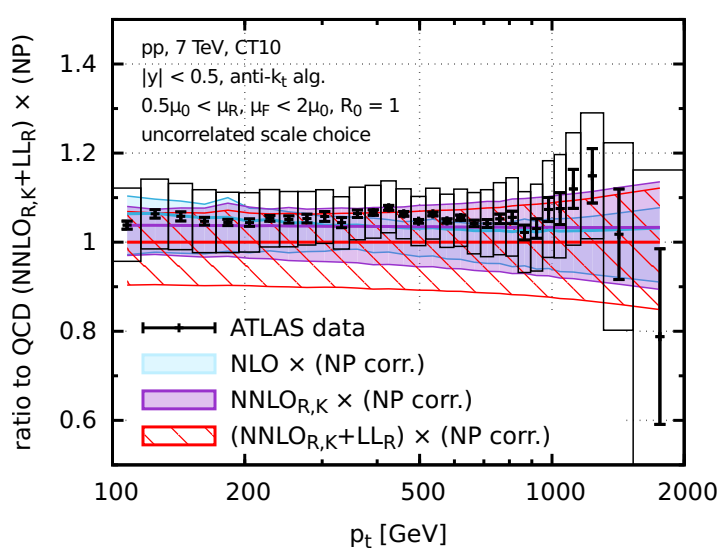

inclusive jets, $\mathrm{R}=0.6, \mathrm{~K}=1.10$

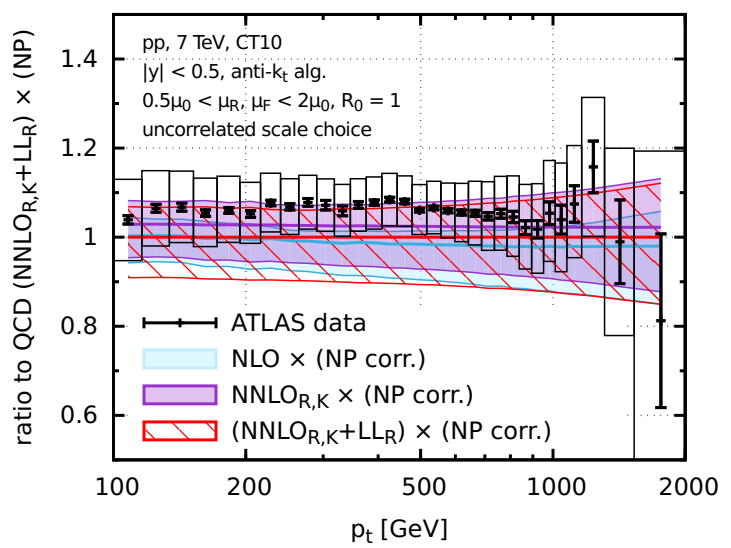

Figure 21. Comparison between theoretical predictions with a NNLO $R_{m}=1$ correction factor $K=1.10$ and data from ATLAS at $\sqrt{s}=7 \mathrm{TeV}[27]$ in the rapidity bin $|y|<0.5$, for $R=0.4$ and $R=0.6$. Rectangular boxes indicate the size of systematic uncertainties on the data points, while the errors bars correspond to the statistical uncertainties. Results are normalised to the central $\mathrm{NNLO}_{R, K}+\mathrm{LL}_{R}$ prediction (including non-perturbative corrections).

inclusive jets, $\mathrm{R}=0.4, \mathrm{~K}=1.10$

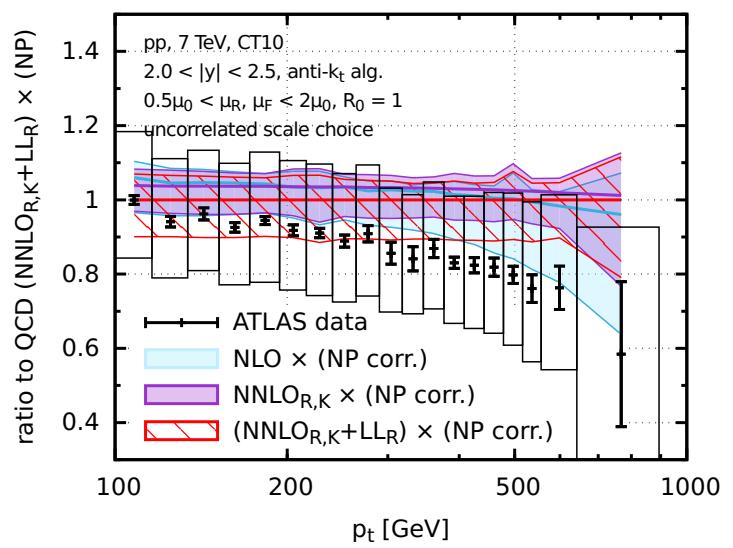

inclusive jets, $\mathrm{R}=0.6, \mathrm{~K}=1.10$

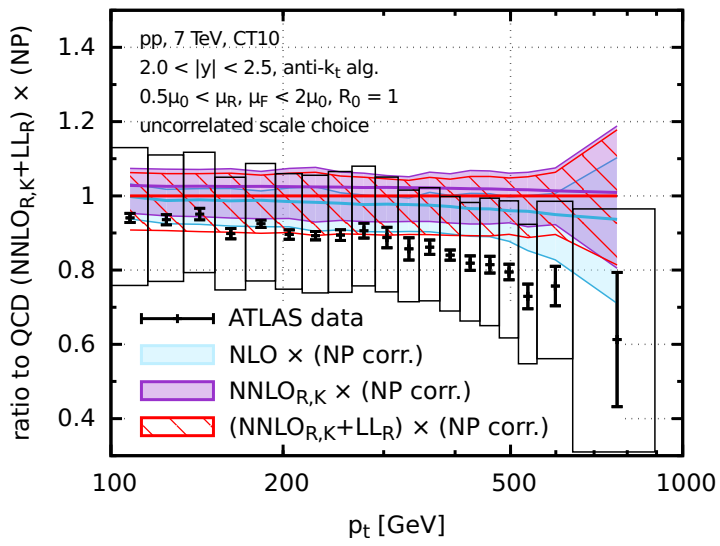

Figure 22. Analogue of figure 21, but for the rapidity bin $2<|y|<2.5$.

We also saw that there are large $R$-dependent terms at NNLO that are beyond the control of our $\mathrm{LL}_{R}$ resummation (sections 2.2 and 4). To account for them in the absence of the full NNLO calculation, we introduced a stand-in for NNLO that we called $\mathrm{NNLO}_{R}$. This is defined to be identical to NLO for $R=1$ but includes full NNLO $R$ dependence, which can be obtained from a NLO 3-jet calculation. Once complete NNLO predictions become available, it will be trivial to replace the $\mathrm{NNLO}_{R}$ terms with NNLO ones.

For an accurate description of the inclusive jet spectrum one must also account for nonperturbative effects. In section 6 we revisited the analytical hadronisation predictions of 
ref. [20]. We found that the predicted scaling with $R$ and the parton flavour was consistent with what is observed in Monte Carlo simulations. However such simulations additionally show a non-trivial $p_{t}$ dependence that is absent from simple analytical estimates. Accordingly we decided to rely just on Monte Carlo simulations to evaluate non-perturbative corrections.

We compared our results to data from the ALICE and ATLAS collaborations in section 6. For the smallest available $R$ value of 0.2 , both the $\mathrm{NNLO}_{R}$ and the $\mathrm{LL}_{R}$ corrections beyond $\mathrm{NNLO}_{R}$ play important roles and at the lower end of ALICE's $p_{t}$ range, the effect of $\mathrm{NNLO}_{R}$ corrections was almost $50 \%$, while further $\mathrm{LL}_{R}$ corrections mattered at the $20 \%$ level. For $R=0.4, \mathrm{NNLO}_{R}$ corrections still mattered, typically at the $10-30 \%$ level, depending on the $p_{t}$. However $\mathrm{LL}_{R}$ resummation then brought little additional change. Overall, for the ALICE data and the forward ATLAS data, $\mathrm{NNLO}_{R}+\mathrm{LL}_{R}$ brought somewhat better agreement than NLO, while for central rapidities, the ATLAS data were substantially above the $\mathrm{NNLO}_{R}+\mathrm{LL}_{R}$ predictions. It will be important to revisit the pattern of agreement once the full NNLO corrections are known, taking into account also aspects such as correlated experimental systematic uncertainties and PDF uncertainties.

Where the $\mathrm{NNLO}_{R}$ and $\mathrm{NNLO}_{R}+\mathrm{LL}_{R}$ predictions clearly make the most difference is for reproducing the $R$-dependence of the cross sections. For the inclusive spectrum plots, once one goes to $\mathrm{NNLO}_{R}+\mathrm{LL}_{R}$, the picture that emerges is consistent across different values of $R$. That was not the case at NLO. This is visible also in the ratios of cross sections at different $R$ values. In particular, for the reasonably precise ATLAS data, $\mathrm{NNLO}_{R}$ and $\mathrm{NNLO}+\mathrm{LL}_{R}$ are in much better agreement with the data than the NLO-based predictions. For the ALICE data, the uncertainties are such that it is harder to make a definitive statement. Nevertheless $\mathrm{NNLO}+\mathrm{LL}_{R}$ performs well and notably better than plain $\mathrm{NNLO}_{R}$.

Overall, the substantial size of subleading $R$-enhanced terms in the NNLO corrections also motivates studies of small- $R$ resummation beyond $L_{L}$ accuracy and of small- $R$ higher order effects in other jet observables.

A final comment concerns long-term prospects. We have seen here that the availability of data at multiple $R$ values provides a powerful handle to cross-check theoretical predictions. As the field moves towards ever higher precision, with improved theoretical predictions and reduced experimental systematic uncertainties, cross checks at multiple $R$ values will, we believe, become increasingly important. In this respect, we strongly encourage measurements at three different radii. Small radii, $R \simeq 0.2-0.3$, are particularly sensitive to hadronisation effects; large radii, $R \simeq 0.6-0.8$ to underlying event effects; the use of an intermediate radius $R \simeq 0.4$ minimises both and provides a good central choice. Only with the use of three radii do we have a realistic chance of disentangling the three main sources of theoretical uncertainties, namely perturbative effects, hadronisation, the underlying event.

\section{Acknowledgments}

MD and GPS are grateful to the MITP for hospitality and support during part of this work. We would all like to thank Matteo Cacciari for numerous useful discussions and comments throughout this work. We are grateful to Simone Alioli and Emanuele Re for 

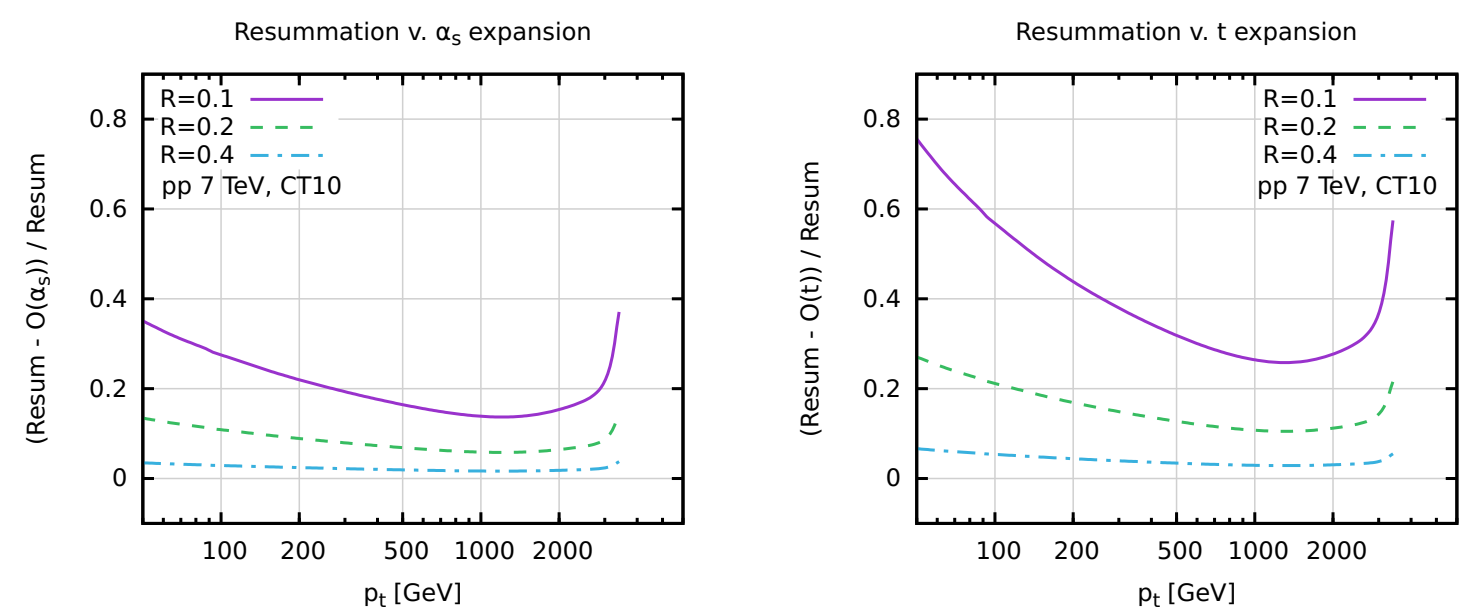

Figure 23. Comparison of the convergence of the $\alpha_{s}$ expansion of the small- $R$ resummation (left) relative to that of the $t$ expansion (right).

assistance and cross-checks with POWHEG and to them and the remaining authors of ref. [44] for discussions about the results. We thank Peter Skands for discussions about the nonperturbative effects. We also thank Jan Kretzschmar, Mark Sutton and Pavel Starovoitov for discussions about ATLAS's non-perturbative correction factors and the first of them for discussions about the determination of uncertainties on ratios of cross sections at different radius values. Finally we are grateful to an anonymous referee for constructive comments on the structure of the paper.

MD's work is supported in part by the Lancaster-Manchester-Sheffield Consortium for Fundamental Physics under STFC grant ST/L000520/1. FD is supported by the ILP LABEX (ANR-10-LABX-63) supported by French state funds managed by the ANR within the Investissements d'Avenir programme under reference ANR-11-IDEX-0004-02. GPS and GS's work is supported by ERC advanced grant Higgs@LHC. GS's work is also supported by the Paris-Saclay IDEX.

\section{A Differences in $\alpha_{s}$ and $t$ expansions}

In our original work on small- $R$ effects [7], we compared the $L_{R}$ resummed results to an expansion in powers of $t$, with $t$ as defined in eq. (2.2). However $t$ is a non-trivial function of $\alpha_{s}$ and expansions in $\alpha_{s}$ and $t$ can have different convergence properties.

This is illustrated in figure 23. The left-hand plot shows the difference between the resummation and its expansion to NLO in powers of $\alpha_{s}$, normalised to the full resummed result. The right-hand plot shows the difference between the resummation and its expansion to NLO in powers of $t$, with the same normalisation. Three different $R$ values are shown. One sees that the $t$ expansion converges more slowly than the $\alpha_{s}$ expansion.

At first sight, this observation is somewhat surprising, insofar as $t$ would appear to be the natural variable for considering small $R$ effects. Part of the explanation is as 
follows: the $t$ expansion has alternating sign coefficients, at least for the first couple of orders. This means that the NLO $\mathcal{O}(t)$ correction (relative to LO) is larger than the overall effect of resummation. The $\mathcal{O}\left(\alpha_{s}\right)$ correction has the same coefficient as the $\mathcal{O}(t)$ correction (modulo an overall normalisation factor of $\frac{1}{2 \pi} \ln R_{0}^{2} / R^{2}$ ). However, $t$ involves the average of the coupling over a range of scales between $p_{t}$ and $R p_{t}$, which is larger than $\alpha_{s}\left(p_{t}\right)$. Consequently, the NLO $\mathcal{O}\left(\alpha_{s}\right)$ term is not as large as the NLO $\mathcal{O}(t)$ term, and it overshoots the resummation by less. Though not illustrated in figure 23, a similar phenomenon occurs also when comparing to expansions at NNLO.

One should keep in mind that for observables other than the inclusive jet spectrum, it may no longer be true that a $t$ expansion converges more slowly than an $\alpha_{s}$ expansion. Rather, when discussing fixed-order convergence properties compared to full small- $R$ resummation, one should simply be aware that the convergence properties of the $t$ and $\alpha_{s}$ expansions will be sometimes be noticeably different.

Note also that the above discussion holds specifically for the expansion of the $\mathrm{LL}_{R}$ result. As we have seen in section 2.2, $\mathrm{NLL}_{R}$ effects are large and at NNLO are of opposite sign to the $\mathrm{LL}_{R}$ contribution. This further complicates the discussion of the convergence properties of the inclusive jet spectrum.

\section{B Scale choice beyond leading order}

When making fixed-order predictions for the inclusive jet cross section, there are two widely used prescriptions for the choice of a central renormalisation and factorisation scale. One prescription is to use a single scale for the whole event, set by the $p_{t}$ of the hardest jet in the event, $\mu_{0}=p_{t, \max }$. This was adopted, for example, in ref. [27]. Another prescription is to take instead a different scale for each jet, specifically that jet's $p_{t}, \mu_{0}=p_{t, \text { jet }}$. This was adopted for example in ref. [13]. ${ }^{13}$

At LO, the two prescriptions give identical results, since there are only two jets in the event and they have the same $p_{t}$. However, starting from NLO the prescriptions can differ substantially. Interestingly, a study of the small-radius limit can provide considerable insight into which choice is more appropriate.

Figure 24 (left) shows the ratio of the NLO result as obtained with $\mu_{0}=p_{t, \text { jet }}$ to that with $\mu_{0}=p_{t, \max }$, as a function of the jet $p_{t}$, for three different jet radii. The main observation is that the $\mu_{0}=p_{t, \text { jet }}$ prescription increases the cross section, especially at small radii: it brings an increase of almost $20 \%$ for $R=0.1$ at low $p_{t}$, versus $\lesssim 4 \%$ for $R=1.0$ (in both cases for a central scale choice). As we saw in section 4 , for reasonably small $R$, the NNLO corrections suppress the cross section. Therefore the choice $\mu_{0}=p_{t, \text { jet }}$ takes us in the wrong direction.

In order to understand this better, it is useful to make a number of observations:

1. For the virtual part of the NLO calculation, the two scale prescriptions give identical results, so the deviation of the ratio from 1 in figure 24 (left) can come only from the real part.

\footnotetext{
${ }^{13}$ Note that yet other scale choices have been used in the literature, notably in predictions for dijet masses [48, 75].
} 

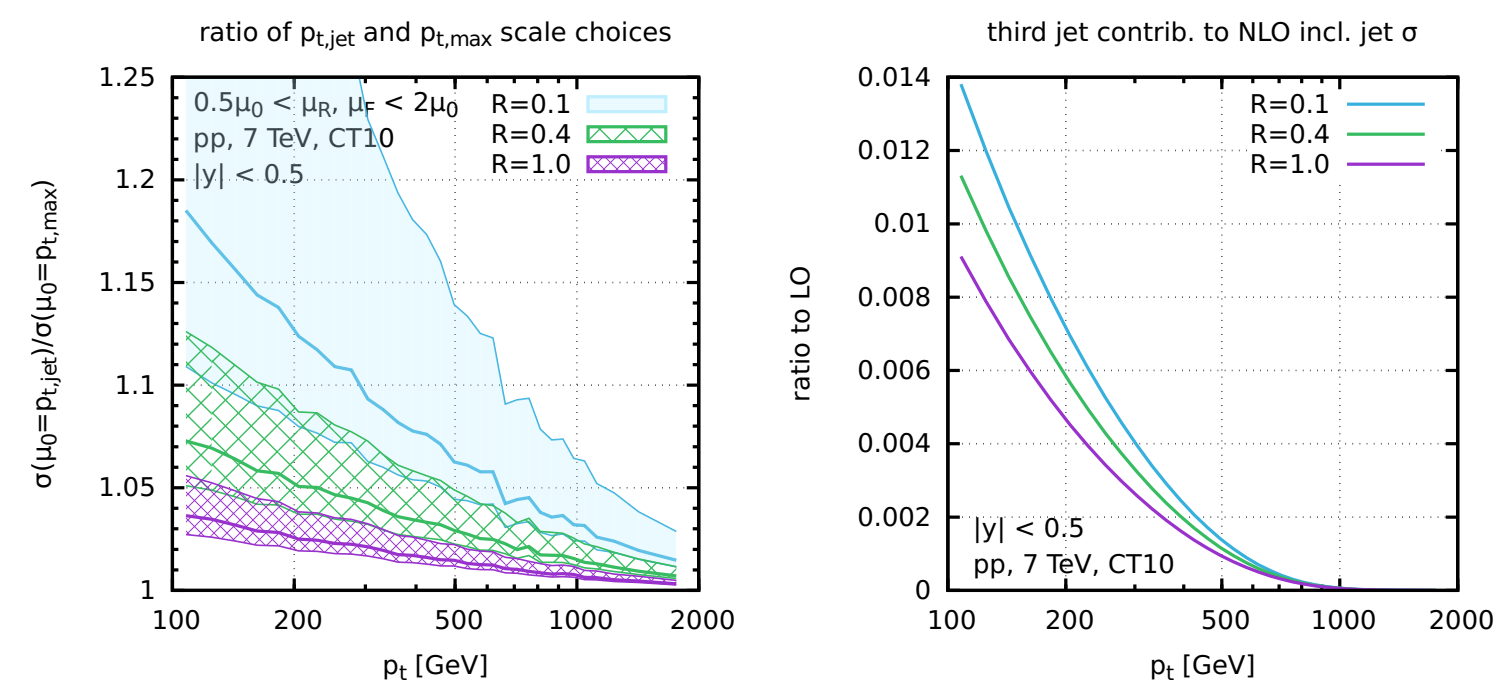

Figure 24. Left: ratio of NLO predictions for the inclusive spectrum when using the per-jet scale choice $\mu_{0}=p_{t, \text { jet }}$ versus the per-event choice $\mu_{0}=p_{t, \max }$. The results are shown as a function of jet $p_{t}$ for three jet radius choices, $R=0.1,0.4$ and 1.0 and have been obtained with NLOJet++. The bands correspond to the effect of scale variation, where the scales are varied upwards and downwards by a factor of two simultaneously for the numerator and denominator. Right: fraction of the inclusive jet spectrum (for $|y|<0.5$ ) that comes from jets beyond the two hardest. The 3 -jet rate and the overall normalisation are both evaluated at LO.

2. The real part itself involves two different pieces: that from binning either of the two leading jets, and that from binning the 3rd jet. The right-hand plot of figure 24 shows that the leading-order 3rd-jet contribution is at the level of $1-2 \%$ of the leading-order dijet result and so it is reasonable to neglect it in our discussion. ${ }^{14}$

3. When a real emission is within an angle $R$ of its nearest other parton, there are only two jets in the event and the two scale-choice prescriptions are identical.

4. Differences between the prescriptions arise when the softest parton falls outside one of the two leading jets. Then one of those jets has a reduced $p_{t}$ and the choice $\mu_{0}=p_{t, \text { jet }}$ gives a smaller scale than $\mu_{0}=p_{t, \max }$. This occurs with a probability that is enhanced by $\ln 1 / R$.

5. At $p_{t} \sim 100 \mathrm{GeV}$, where the effects are largest, renormalisation scale $\left(\mu_{R}\right)$ variations play a much larger role than factorisation scale $\left(\mu_{F}\right)$ variations. Therefore a smaller scale translates to a larger value of $\alpha_{s}$ and thus a larger cross section for the real contribution (which is always positive). Consequently, the prescription $\mu_{0}=p_{t, \text { jet }}$ leads to a cross section that is larger than the prescription $\mu_{0}=p_{t, \max }$ and the difference is enhanced by a factor $\ln 1 / R$ for small $R$.

\footnotetext{
${ }^{14}$ The 3 rd jet is produced with a probability $\mathcal{O}\left(\alpha_{s}\right)$, however because its $p_{t}$ is lower than that of the two leading jets, its contribution to the (steeply falling) jet spectrum is substantially suppressed.
} 


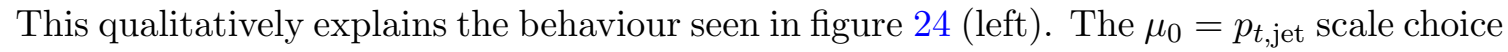
introduces a correction that goes in the wrong direction because it leads to a smaller scale (and larger $\alpha_{s}$ ) for the real part, but without a corresponding modification of the virtual part. Thus it breaks the symmetry between real and virtual corrections.

The above reasoning leads us to prefer the $\mu_{0}=p_{t, \max }^{R=1}$ prescription. To make it a unique event-wide choice, independent of $R$, we define always define $\mu_{0}=p_{t, \text { max }}$ using jets with a radius equal to one, regardless of the $R$ value used in the measurement.

We note that $\mu_{0}=p_{t, \max }$ has a potential linear sensitivity to initial-state radiation, i.e. initial state radiation of transverse momentum $p_{t, i}$ shifts $\mu_{0}$ by an amount $p_{t, i}$. A yet more stable choice might be $\mu_{0}=\frac{1}{2}\left(p_{t, 1}+p_{t, 2}\right)$, the average transverse momentum of the two hardest jets (again defined with a radius of one). For this choice, the shift of $\mu_{0}$ would be limited $\mathcal{O}\left(p_{t, i}^{2} /\left(p_{t, 1}+p_{t, 2}\right)\right)$. We leave its study to future work.

Yet another option is the use of MINLO type procedures [76]. For dijet systems, this should be rather similar to $\mu_{0}=\frac{1}{2}\left(p_{t, 1}+p_{t, 2}\right)$.

Open Access. This article is distributed under the terms of the Creative Commons Attribution License (CC-BY 4.0), which permits any use, distribution and reproduction in any medium, provided the original author(s) and source are credited.

\section{References}

[1] F. Aversa, L. Gonzales, M. Greco, P. Chiappetta and J.P. Guillet, Jet cone size dependence of inclusive cross-sections to $O\left(\alpha_{s}^{3}\right), Z$. Phys. C 49 (1991) 459 [InSPIRE].

[2] F. Aversa, M. Greco, P. Chiappetta and J.P. Guillet, Jet inclusive production to $O\left(\alpha_{s}^{3}\right)$ : comparison with data, Phys. Rev. Lett. 65 (1990) 401 [INSPIRE].

[3] J.P. Guillet, One jet $O\left(\alpha_{s}^{3}\right)$ cross-section: mass dependence, Z. Phys. C 51 (1991) 587 [INSPIRE].

[4] B. Jager, M. Stratmann and W. Vogelsang, Single inclusive jet production in polarized pp collisions at $O\left(\right.$ alpha $\left._{s}^{3}\right)$, Phys. Rev. D 70 (2004) 034010 [hep-ph/0404057] [INSPIRE].

[5] A. Mukherjee and W. Vogelsang, Jet production in (un)polarized pp collisions: dependence on jet algorithm, Phys. Rev. D 86 (2012) 094009 [arXiv:1209.1785] [INSPIRE].

[6] T. Kaufmann, A. Mukherjee and W. Vogelsang, Next-to-leading order calculation for jets defined by a maximized jet function, Phys. Rev. D 91 (2015) 034001 [arXiv:1412.0298] [INSPIRE].

[7] M. Dasgupta, F. Dreyer, G.P. Salam and G. Soyez, Small-radius jets to all orders in QCD, JHEP 04 (2015) 039 [arXiv: 1411.5182] [INSPIRE].

[8] S. Catani, M. Fontannaz, J.P. Guillet and E. Pilon, Isolating prompt photons with narrow cones, JHEP 09 (2013) 007 [arXiv: 1306.6498] [INSPIRE].

[9] S. Alioli and J.R. Walsh, Jet veto clustering logarithms beyond leading order, JHEP 03 (2014) 119 [arXiv:1311.5234] [INSPIRE].

[10] T. Becher, M. Neubert, L. Rothen and D.Y. Shao, Effective field theory for jet processes, Phys. Rev. Lett. 116 (2016) 192001 [arXiv: 1508.06645] [INSPIRE]. 
[11] Y.-T. Chien, A. Hornig and C. Lee, Soft-collinear mode for jet cross sections in soft collinear effective theory, Phys. Rev. D 93 (2016) 014033 [arXiv:1509.04287] [INSPIRE].

[12] A. Banfi et al., Jet-vetoed Higgs cross section in gluon fusion at $N^{3} L O+N N L L$ with small- $R$ resummation, JHEP 04 (2016) 049 [arXiv: 1511.02886] [INSPIRE].

[13] ALICE collaboration, Measurement of the inclusive differential jet cross section in pp collisions at $\sqrt{s}=2.76 \mathrm{TeV}$, Phys. Lett. B 722 (2013) 262 [arXiv:1301.3475] [INSPIRE].

[14] CMS collaboration, Measurement of the ratio of inclusive jet cross sections using the anti- $k_{T}$ algorithm with radius parameters $R=0.5$ and 0.7 in pp collisions at $\sqrt{s}=7 \mathrm{TeV}$, Phys. Rev. D 90 (2014) 072006 [arXiv:1406.0324] [INSPIRE].

[15] STAR collaboration, M. Ploskon, Inclusive cross section and correlations of fully reconstructed jets in $\sqrt{s_{\mathrm{NN}}}=200 \mathrm{GeV}$ Au+Au and $p+p$ collisions, Nucl. Phys. A 830 (2009) 255C [arXiv:0908.1799] [INSPIRE].

[16] S. Eckweiler, Measurement of the inclusive jet cross-section in proton-proton collisions at $\sqrt{s}=7 \mathrm{TeV}$ with the ATLAS detector, Ph.D. thesis, CERN-THESIS-2011-145, Mainz U., Mainz Germany (2011).

[17] G. Soyez, A simple description of jet cross-section ratios, Phys. Lett. B 698 (2011) 59 [arXiv:1101.2665] [INSPIRE].

[18] J. Currie, A. Gehrmann-De Ridder, E.W.N. Glover and J. Pires, NNLO QCD corrections to jet production at hadron colliders from gluon scattering, JHEP 01 (2014) 110 [arXiv: 1310.3993] [INSPIRE].

[19] J. Pires, Future prospects of $\alpha_{s}$ from jets at the LHC at NNLO, talk at the Workshop on high-precision $\alpha_{s}$ measurements: from LHC to FCC-ee, CERN, Geneva Switzerland October 2015.

[20] M. Dasgupta, L. Magnea and G.P. Salam, Non-perturbative QCD effects in jets at hadron colliders, JHEP 02 (2008) 055 [arXiv:0712.3014] [INSPIRE].

[21] T. Sjöstrand, S. Mrenna and P.Z. Skands, PYTHIA 6.4 physics and manual, JHEP 05 (2006) 026 [hep-ph/0603175] [inSPIRE].

[22] T. Sjöstrand, S. Mrenna and P.Z. Skands, A brief introduction to PYTHIA 8.1, Comput. Phys. Commun. 178 (2008) 852 [arXiv:0710.3820] [INSPIRE].

[23] G. Corcella et al., HERWIG 6: an event generator for hadron emission reactions with interfering gluons (including supersymmetric processes), JHEP 01 (2001) 010 [hep-ph/0011363] [INSPIRE].

[24] G. Corcella et al., HERWIG 6.5 release note, hep-ph/0210213 [INSPIRE].

[25] M. Bahr et al., HERWIG++ physics and manual, Eur. Phys. J. C 58 (2008) 639 [arXiv: 0803.0883] [INSPIRE].

[26] J. Bellm et al., HERWIG++ 2.7 release note, arXiv:1310.6877 [INSPIRE].

[27] ATLAS collaboration, Measurement of the inclusive jet cross-section in proton-proton collisions at $\sqrt{s}=7 \mathrm{TeV}$ using $4.5 \mathrm{fb}^{-1}$ of data with the ATLAS detector, JHEP 02 (2015) 153 [Erratum ibid. 09 (2015) 141] [arXiv:1410.8857] [INSPIRE].

[28] M. Dasgupta, F.A. Dreyer, G.P. Salam and G. Soyez, MicroJets webpage, http://microjets.hepforge.org. 
[29] R.K. Ellis, W.J. Stirling and B.R. Webber, QCD and collider physics, Camb. Monogr. Part. Phys. Nucl. Phys. Cosmol. 8 (1996) 1 [InSPIRE].

[30] S. Catani, Y.L. Dokshitzer, M.H. Seymour and B.R. Webber, Longitudinally invariant $K_{t}$ clustering algorithms for hadron hadron collisions, Nucl. Phys. B 406 (1993) 187 [INSPIRE].

[31] S.D. Ellis and D.E. Soper, Successive combination jet algorithm for hadron collisions, Phys. Rev. D 48 (1993) 3160 [hep-ph/9305266] [INSPIRE].

[32] Y.L. Dokshitzer, G.D. Leder, S. Moretti and B.R. Webber, Better jet clustering algorithms, JHEP 08 (1997) 001 [hep-ph/9707323] [INSPIRE].

[33] M. Wobisch and T. Wengler, Hadronization corrections to jet cross-sections in deep inelastic scattering, in Monte Carlo generators for HERA physics, Hamburg Germany 1998/1999, pg. 270 [hep-ph/9907280] [INSPIRE].

[34] M. Cacciari, G.P. Salam and G. Soyez, The anti-k $k_{t}$ jet clustering algorithm, JHEP 04 (2008) 063 [arXiv: 0802.1189] [INSPIRE].

[35] G.P. Salam and G. Soyez, A practical seedless infrared-safe cone jet algorithm, JHEP 05 (2007) 086 [arXiv:0704.0292] [INSPIRE].

[36] M. Cacciari, G.P. Salam and G. Soyez, FastJet user manual, Eur. Phys. J. C 72 (2012) 1896 [arXiv: 1111.6097] [INSPIRE].

[37] G.P. Salam and J. Rojo, A higher order perturbative parton evolution toolkit (HOPPET), Comput. Phys. Commun. 180 (2009) 120 [arXiv:0804.3755] [InSPIRE].

[38] Z. Nagy, Next-to-leading order calculation of three jet observables in hadron hadron collision, Phys. Rev. D 68 (2003) 094002 [hep-ph/0307268] [InSPIRE].

[39] H.-L. Lai et al., New parton distributions for collider physics, Phys. Rev. D 82 (2010) 074024 [arXiv: 1007.2241] [INSPIRE].

[40] A. Gehrmann-De Ridder, T. Gehrmann, E.W.N. Glover, A. Huss and T.A. Morgan, NNLO $Q C D$ corrections for $Z$ boson plus jet production, arXiv:1601.04569 [INSPIRE].

[41] I.W. Stewart and F.J. Tackmann, Theory uncertainties for Higgs and other searches using jet bins, Phys. Rev. D 85 (2012) 034011 [arXiv:1107.2117] [INSPIRE].

[42] A. Banfi, G.P. Salam and G. Zanderighi, $N L L+N N L O$ predictions for jet-veto efficiencies in Higgs-boson and Drell-Yan production, JHEP 06 (2012) 159 [arXiv:1203.5773] [INSPIRE].

[43] D. de Florian, P. Hinderer, A. Mukherjee, F. Ringer and W. Vogelsang, Approximate next-to-next-to-leading order corrections to hadronic jet production, Phys. Rev. Lett. 112 (2014) 082001 [arXiv:1310.7192] [INSPIRE].

[44] S. Alioli, K. Hamilton, P. Nason, C. Oleari and E. Re, Jet pair production in POWHEG, JHEP 04 (2011) 081 [arXiv: 1012.3380] [INSPIRE].

[45] R. Corke and T. Sjöstrand, Interleaved parton showers and tuning prospects, JHEP 03 (2011) 032 [arXiv:1011.1759] [INSPIRE].

[46] P.Z. Skands, Tuning Monte Carlo generators: the Perugia tunes, Phys. Rev. D 82 (2010) 074018 [arXiv: 1005.3457 ] [INSPIRE].

[47] ATLAS collaboration, New ATLAS event generator tunes to 2010 data, ATL-PHYS-PUB-2011-008, CERN, Geneva Switzerland (2011) [ATL-COM-PHYS-2011-329]. 
[48] ATLAS collaboration, Measurement of inclusive jet and dijet production in pp collisions at $\sqrt{s}=7 \mathrm{TeV}$ using the ATLAS detector, Phys. Rev. D 86 (2012) 014022 [arXiv:1112.6297] [INSPIRE].

[49] Y.L. Dokshitzer and B.R. Webber, Calculation of power corrections to hadronic event shapes, Phys. Lett. B 352 (1995) 451 [hep-ph/9504219] [INSPIRE].

[50] G.P. Korchemsky and G.F. Sterman, Nonperturbative corrections in resummed cross-sections, Nucl. Phys. B 437 (1995) 415 [hep-ph/9411211] [INSPIRE].

[51] M.H. Seymour, Jet shapes in hadron collisions: higher orders, resummation and hadronization, Nucl. Phys. B 513 (1998) 269 [hep-ph/9707338] [INSPIRE].

[52] M. Cacciari, G.P. Salam and G. Soyez, The catchment area of jets, JHEP 04 (2008) 005 [arXiv: 0802.1188] [INSPIRE].

[53] Y.L. Dokshitzer, G. Marchesini and B.R. Webber, Dispersive approach to power behaved contributions in QCD hard processes, Nucl. Phys. B 469 (1996) 93 [hep-ph/9512336] [INSPIRE].

[54] P. Skands, S. Carrazza and J. Rojo, Tuning PYTHIA 8.1: the Monash 2013 tune, Eur. Phys. J. C 74 (2014) 3024 [arXiv: 1404.5630] [INSPIRE].

[55] R. Field, Early LHC underlying event data - findings and surprises, arXiv:1010.3558 [INSPIRE].

[56] B. Cooper, J. Katzy, M.L. Mangano, A. Messina, L. Mijovic and P. Skands, Importance of a consistent choice of $\alpha_{s}$ in the matching of ALPGEN and PYTHIA, Eur. Phys. J. C 72 (2012) 2078 [arXiv: 1109.5295] [INSPIRE].

[57] G.P. Salam and D. Wicke, Hadron masses and power corrections to event shapes, JHEP 05 (2001) 061 [hep-ph/0102343] [INSPIRE].

[58] V. Mateu, I.W. Stewart and J. Thaler, Power corrections to event shapes with mass-dependent operators, Phys. Rev. D 87 (2013) 014025 [arXiv:1209.3781] [INSPIRE].

[59] ATLAS collaboration, Study of jet shapes in inclusive jet production in pp collisions at $\sqrt{s}=7$ TeV using the ATLAS detector, Phys. Rev. D 83 (2011) 052003 [arXiv:1101.0070] [INSPIRE].

[60] CMS collaboration, Shape, transverse size and charged hadron multiplicity of jets in $p p$ collisions at $7 \mathrm{TeV}$, JHEP 06 (2012) 160 [arXiv:1204.3170] [INSPIRE].

[61] G.P. Korchemsky and G.F. Sterman, Power corrections to event shapes and factorization, Nucl. Phys. B 555 (1999) 335 [hep-ph/9902341] [INSPIRE].

[62] S. Gieseke, C. Rohr and A. Siodmok, Colour reconnections in HERWIG++, Eur. Phys. J. C 72 (2012) 2225 [arXiv:1206.0041] [INSPIRE].

[63] J. Collins and J.-W. Qiu, $k_{T}$ factorization is violated in production of high-transverse-momentum particles in hadron-hadron collisions, Phys. Rev. D 75 (2007) 114014 [arXiv: 0705.2141] [INSPIRE].

[64] CMS collaboration, Measurements of differential jet cross sections in proton-proton collisions at $\sqrt{s}=7 \mathrm{TeV}$ with the CMS detector, Phys. Rev. D 87 (2013) 112002 [Erratum ibid. D 87 (2013) 119902] [arXiv: 1212.6660] [INSPIRE].

[65] CMS collaboration, Measurement of the inclusive jet cross section in pp collisions at $\sqrt{s}=2.76 \mathrm{TeV}$, Eur. Phys. J. C 76 (2016) 265 [arXiv: 1512.06212] [INSPIRE]. 
[66] J. Gao et al., CT10 next-to-next-to-leading order global analysis of QCD, Phys. Rev. D 89 (2014) 033009 [arXiv: 1302.6246] [INSPIRE].

[67] S. Dulat et al., New parton distribution functions from a global analysis of quantum chromodynamics, Phys. Rev. D 93 (2016) 033006 [arXiv: 1506.07443] [INSPIRE].

[68] A.D. Martin, W.J. Stirling, R.S. Thorne and G. Watt, Parton distributions for the LHC, Eur. Phys. J. C 63 (2009) 189 [arXiv:0901.0002] [inSPIRE].

[69] L.A. Harland-Lang, A.D. Martin, P. Motylinski and R.S. Thorne, Parton distributions in the LHC era: MMHT 2014 PDFs, Eur. Phys. J. C 75 (2015) 204 [arXiv:1412.3989] [InSPIRE].

[70] NNPDF collaboration, R.D. Ball et al., Parton distributions for the LHC run II, JHEP 04 (2015) 040 [arXiv: 1410.8849] [INSPIRE].

[71] S. Dittmaier, A. Huss and C. Speckner, Weak radiative corrections to dijet production at hadron colliders, JHEP 11 (2012) 095 [arXiv:1210.0438] [INSPIRE].

[72] U. Baur, Weak boson emission in hadron collider processes, Phys. Rev. D 75 (2007) 013005 [hep-ph/0611241] [INSPIRE].

[73] N. Méric, Etude théorique et expérimentale des corrections électrofaibles au processus de production inclusive de jets. Développement de méthodes de détection de topologies extrêmes (in French), Ph.D. thesis, CERN-THESIS-2013-358, https://tel.archives-ouvertes.fr/tel-00959427/, Diderot U., Paris France (2013).

[74] ATLAS collaboration, HepData entry corresponding to ref. [27], http://hepdata.cedar.ac.uk/view/ins1325553.

[75] S.D. Ellis, Z. Kunszt and D.E. Soper, Two jet production in hadron collisions at order $\alpha_{s}^{3}$ in QCD, Phys. Rev. Lett. 69 (1992) 1496 [INSPIRE].

[76] K. Hamilton, P. Nason and G. Zanderighi, MINLO: multi-scale improved NLO, JHEP 10 (2012) 155 [arXiv: 1206.3572] [INSPIRE]. 